\title{
Induced Pluripotent Stem Cells in Dental and Nondental Tissue Regeneration: A Review of an Unexploited Potential
}

\author{
Israa Ahmed Radwan $\mathbb{D}^{1,},{ }^{1,2}$ Dina Rady, ${ }^{1,2}$ Marwa M. S. Abbass, ${ }^{1,2}$ Sara El Moshy, ${ }^{1,2}$ \\ Nermeen AbuBakr $\mathbb{D}^{1},{ }^{1,2}$ Christof E. Dörfer, ${ }^{3}$ and Karim M. Fawzy El-Sayed $\mathbb{D}^{2,3,4}$ \\ ${ }^{1}$ Oral Biology Department, Faculty of Dentistry, Cairo University, Cairo, Egypt \\ ${ }^{2}$ Stem Cells and Tissue Engineering Research Group, Faculty of Dentistry, Cairo University, Cairo, Egypt \\ ${ }^{3}$ Clinic for Conservative Dentistry and Periodontology, School of Dental Medicine, Christian Albrechts University, Kiel, Germany \\ ${ }^{4}$ Oral Medicine and Periodontology Department, Faculty of Dentistry, Cairo University, Cairo, Egypt
}

Correspondence should be addressed to Karim M. Fawzy El-Sayed; karim.fawzy@gmail.com

Received 5 December 2019; Accepted 6 March 2020; Published 29 March 2020

Guest Editor: Alireza Moshaverinia

Copyright (C) 2020 Israa Ahmed Radwan et al. This is an open access article distributed under the Creative Commons Attribution License, which permits unrestricted use, distribution, and reproduction in any medium, provided the original work is properly cited.

\begin{abstract}
Cell-based therapies currently represent the state of art for tissue regenerative treatment approaches for various diseases and disorders. Induced pluripotent stem cells (iPSCs), reprogrammed from adult somatic cells, using vectors carrying definite transcription factors, have manifested a breakthrough in regenerative medicine, relying on their pluripotent nature and ease of generation in large amounts from various dental and nondental tissues. In addition to their potential applications in regenerative medicine and dentistry, iPSCs can also be used in disease modeling and drug testing for personalized medicine. The current review discusses various techniques for the production of iPSC-derived osteogenic and odontogenic progenitors, the therapeutic applications of iPSCs, and their regenerative potential in vivo and in vitro. Through the present review, we aim to explore the potential applications of iPSCs in dental and nondental tissue regeneration and to highlight different protocols used for the generation of different tissues and cell lines from iPSCs.
\end{abstract}

\section{Introduction}

Embryonic stem (ES) cells are pluripotent cells derived from the inner cell mass of the blastocyst. They can give rise to tissues derived from the three germ layers and are regarded as a renewable potent cell source for the regeneration of all body tissues [1-4]. However, ES usage in regenerative medicine faces a lot of obstacles as their isolation requires destruction of human embryos which raises justified ethical objections. ES can also elicit an immune response upon transplantation in patients [5]. In 2006, Takahashi et al. [6] demonstrated that mature differentiated cells can be reprogrammed and dedifferentiated into embryonic-like cells, with ES-like properties. Mature murine fibroblast cell lines were reversed into pluripotency via retroviral transduction of 4 transcription factors, POU domain class 5 transcription factor 1 (Oct3/4), the sex-determining region Y-box2 (Sox2),
Kruppel-like factor 4 (Klf4), and myelocytomatosis oncogene (c-Myc), giving rise to induced pluripotent stem cells (iPSCs). Those four transcription factors (also referred to as OSKM factors) were postulated to be responsible for the maintenance of ES inherent pluripotency. Over the subsequent years, iPSCs were generated from a variety of adult tissues [7-9] and were similar to ES in morphology, proliferative rates, surface antigens, expressed genes, and in vivo teratoma formation [6].

\section{2. iPSC Source and Generation (Reprogramming) Methods}

iPSCs were successfully generated from different dental and nondental tissues (Figure 1) including fibroblasts, keratinocytes, melanocyte blood cells, bone marrow cells, adipose cells, tissue-resident progenitor cells, and gingival and 


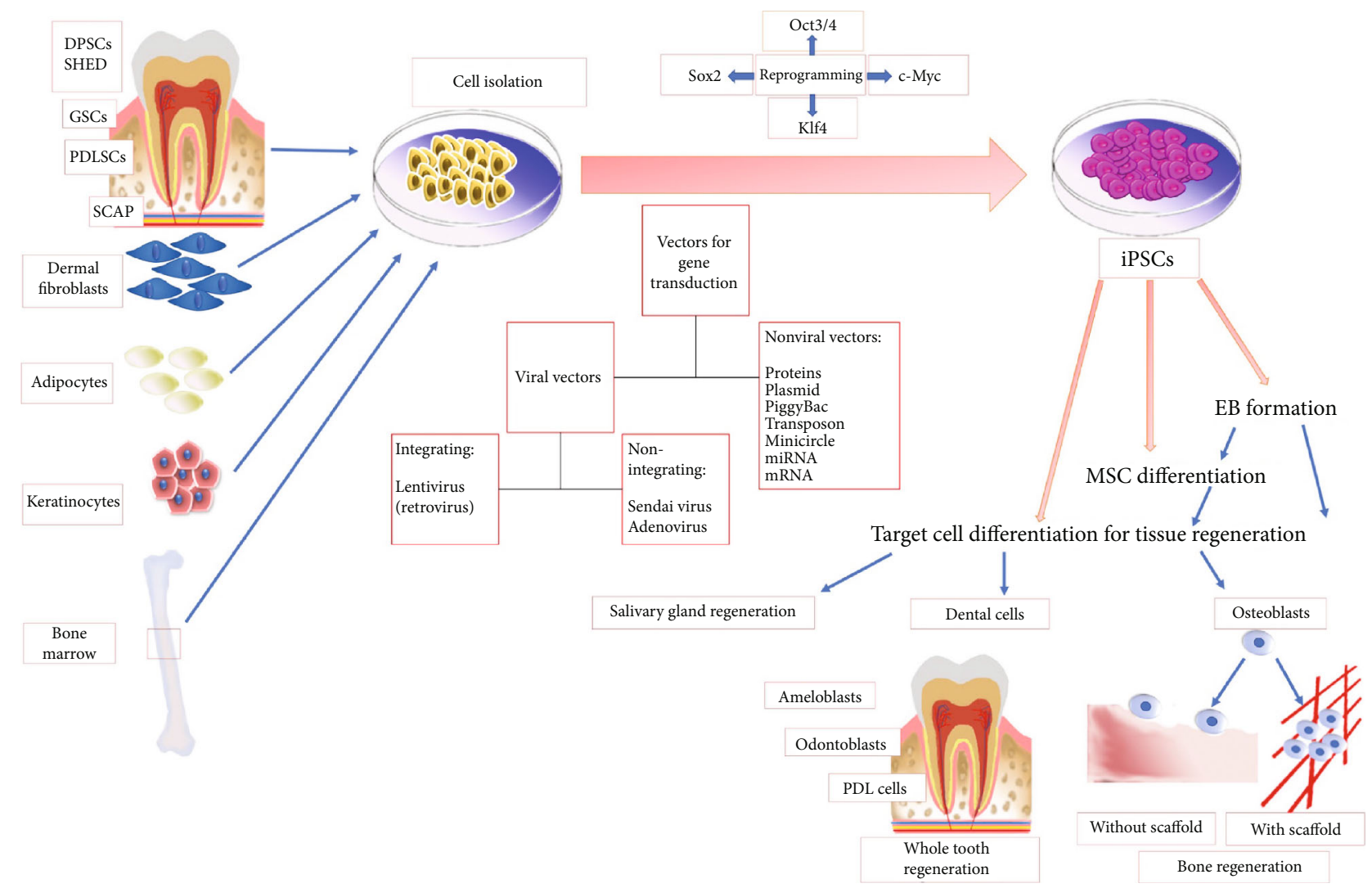

FIGURE 1: Diagram summarizing iPSC source, methods of gene transduction, and iPSC differentiation. Dental pulp stem cells (DPSCs), stem cells from exfoliated deciduous teeth (SHED), gingival stem cells (GSCs), stem cells from apical dental papilla (SCAP), embryoid bodies (EB), mesenchymal stem cells (MSCs), and induced pluripotent stem cells (iPSCs).

periodontal ligament fibroblasts [10-13] via transduction of Oct3/4, Sox2, and Klf4 [14, 15]. iPSCs were also successfully generated from dental pulp stem cells (DPSCs) [16-18], stem cells from human exfoliated deciduous teeth (SHED) $[18,19]$, and stem cells from apical dental papilla [18]. Gingival fibroblast-derived iPSCs were considered to be advantageous over dermal fibroblasts (DF) as they could be easily acquired during routine dental treatment and were effectively reprogrammed into iPSCs [14].

As mentioned above, generation of iPSCs depends on the transduction of specific transcription factors into the somatic cell genome via vectors for its reprogramming [20]. Vectors used during the generation of iPSCs can be divided into integrative viral vectors, integrative free vectors, and nonviral vectors [21]. Originally, lentivirus (a retrovirus), an integrating viral vector, was used for iPSC generation with high reprogramming efficacy [6]. Despite offering a high transduction ability, integrating viral vectors insert their whole genome into recipient cells and may introduce oncogenes or genetic mutations into the host cells [22] (Figure 1).

Nonintegrating viruses, such as Sendai virus and adenovirus, were subsequently introduced in an attempt to overcome these drawbacks [23]. Tashiro et al. [24] compared four types of promoters (RSV, CMV, cytomegalovirus enhancer/b-actin (CA), and elongation factor-1a (EF-1a)) using adenovirus vectors for iPSC induction. An adenovirus vector containing EF-1a and CA promoter efficiently transduced transgenes into mouse iPSCs, without a decrease in pluripotency or viability. An optimized adenovirus vector that was developed by the authors enhanced adipocyte and osteoblast differentiation, confirmed by significant gene expressions of peroxisome proliferator-activated receptor $\mathrm{c}$ and runt-related transcription factor 2 (RUNX2), respectively, by iPSCs.

To avoid an increased risk of tumor generation and chromosomal instability, nonviral vectors were subsequently introduced for the somatic reprogramming process, including proteins, plasmid, piggyBac transposon, minicircle vector, miRNA, and mRNA [25-30]. Gene-editing technologies like CRISPR/Cas9, zinc finger nucleases, and transcription activator-like effector nucleases (TALENs) were additionally employed for genome editing of iPSCs to introduce certain traits for disease modeling and cancer research or to alter their gene expression for possible application in the field of regenerative medicine [31].

\section{Assessment of Pluripotency}

Following iPSC generation, cells have to be assessed via pluripotency assays, including morphological and histological analysis, and certain gene expressions, proving their ability to differentiate into tissues derived from the three germ layers 
and teratoma formation [32]. Teratoma assays involve injection of iPSCs into immunocompromised experimental animals and subsequent formed tissue analysis to assure teratoma formation [33]. Alternatively, in vitro embryoid body (EB) generation can be used to ascertain pluripotency; $\mathrm{EB}$ is a mass of cells derived from all three germ layers [32], generated from iPSCs upon culturing in proper media [32, 34, 35]. EB generation encompasses the homogeneous method as the liquid suspension method and the heterogeneous method as the hanging drop culture. While the heterogeneous method is considered the easiest way to generate EB, the resulting cell masses are largely heterogeneous in size [36], which are irreproducible [37] and negatively affect subsequent iPSC differentiation towards a specific cell line [38]. The homogeneous method, on the other hand, creates cell masses of more homogeneous, uniform sizes that subsequently enhance cell viability and facilitate their subsequent differentiation into specific cell lines $[33,39]$. To avoid tumor formation, prior to implantation, iPSCs are either differentiated into mesenchymal stem cells (MSCs) or targeted tissue cell types with or without EB formation (Figure 1).

\section{4. iPSCs in Dental and Nondental Tissue Regeneration (Table 1)}

4.1. iPSCs and Bone Regeneration. Although autogenous bone graft remains to be the gold standard for reconstruction of bone defects [40], it carries the risk of bone resorption and donor site infection and the graft may not always be available in sufficient amounts [41]. iPSC technologies may provide a suitable alternative to autogenous grafting, whereby patients' somatic cells are induced into bone-forming cells that are loaded on an appropriate scaffold in combination with proper bioactive molecules for bone tissue engineering [42]. To induce osteogenic differentiation of iPSCs, a variety of agents were proposed in isolation or combination, including osteogenic media, ascorbic acid, b-glycerophosphate, dexamethasone, bone morphogenetic proteins (BMPs), and vitamin $\mathrm{D}_{3}$ [43-46]. Osteogenic differentiation is followed by proper characterization of generated bone cells through their expression of osteogenesis-related genes (RUNX2, osteopontin (OPN), osterix (OSX), osteocalcin (OCN), and collagen type I (COL1A1)) [47-50] in addition to the evaluation of in vitro mineralization and alkaline phosphatase (ALP) activity $[51,52]$.

Osteogenic potential of human iPSCs was demonstrated on polymeric nanofibrous polyethersulfone (PES) scaffold with upregulated expressions of osteogenic genes and alkaline phosphatase activity in vitro $[48,53]$. The expression of key osteoblast-related genes in undifferentiated iPSCs was nearly 30 times higher than in undifferentiated ES cells. On the contrary, the expression of the same genes in ES- and iPSC-derived osteoblasts was not significantly different except for OPN and COL1A1, which were significantly higher in iPSC-derived osteoblasts [51]. Evidence revealed that ES cells and iPSCs generated from transgenic mice expressing rat $2.3 \mathrm{~kb}$ type I collagen promoter-driven green fluorescent protein (Col2.3GFP) successfully differentiated into osteoblast lineage cells that expressed Col2.3GFP in vitro [54]. Gene expression profiles proved that ES- and iPSC-derived osteoblasts resemble osteoblasts present in the calvaria [54].

The osteoinductive properties of iPSC-derived bone cells and their capability in treating bone defects were further assessed in vivo by their implantation into a severe combined immunodeficiency (SCID) mouse model. Bone formation was confirmed four weeks following implantation by soft X-ray images [43], X-ray microcomputed tomography $(\mu \mathrm{CT})$ [55], cone beam computed tomography imaging [49], and histological tissue specimens [43, 47-52]. In a cleidocranial dysostosis model, the mutation in RUNX2 gene was repaired in iPSCs derived from mucosal tissues of affected patients. The reverted cells revealed marked upregulation of osteoblast differentiation markers after being cultured in OM for nine days. Loading the differentiated osteoblasts originating from iPSCs with a corrected mutation on a peptide nanofiber scaffold and implanting them into SCID rats' calvarial bone defects revealed reossification four weeks after transplantation with a significant increase in bone volume and bone mineral content [52]. Similarly, osteogenic cells differentiating from EB derived from iPSCs showed positive results in bone regeneration and healing following implantation in the rats' critical-sized calvarial defect $[53,56,57]$ and long bone segmental defect rat model [57] after being loaded on polymeric nanofibrous PES scaffold [53], fibrin glue scaffold [57], hydroxyapatite (HA)/b-tricalcium phosphate scaffold [57], or self-assembling peptide nanofiber hydrogel scaffold [56]. Moreover, iPSCs differentiated into functional osteoblasts and demonstrated a bone regenerative effect comparable to human bone marrow(BM-) MSCs in vivo [57].

4.1.1. Osteogenic Potential of iPSCs-MSCs Obtained through $E B$ Formation. This method entails the differentiation of MSCs from EB-derived iPSCs. It was suggested to possess notable advantages over direct differentiation of iPSCs into osteoblasts, with the resulting osteogenic cells demonstrating a significant upregulation of osteoblast-related genes including ALP, RUNX2, COL1A1, and OCN $[58,59]$. Several factors were demonstrated to influence the osteogenic potential of iPSC-derived MSCs including the incorporation of retinoic acid, transforming growth factor-beta (TGF- $\beta$ ) $[60,61]$, or metformin into the culture media [62] as well as coseeding with other cell types [63-65]. The suspension time of EB and genetic modification of iPSCs-MSCs also proved to affect their osteogenic capability [66-68]. Culturing EB generated from dermal fibroblast iPSCs in media supplemented with TGF- $\beta$ induced MSC differentiation. Two populations of MSCs were recognized, early MSCs that migrated from EB during days 2-5 and late MSCs that crawled from EB during days 5-8. The two iPSC-derived MSC populations and BM-MSCs were transduced with BMP-6 plasmid. Resulting cells were either suspended in fibrin gel and injected into thigh muscles of SCID rats or loaded on collagen scaffolds and implanted in a nonunion radial fracture SCID rat model. No or limited bone formation was acquired upon ectopic injection of BMP-6-late MSCs, 


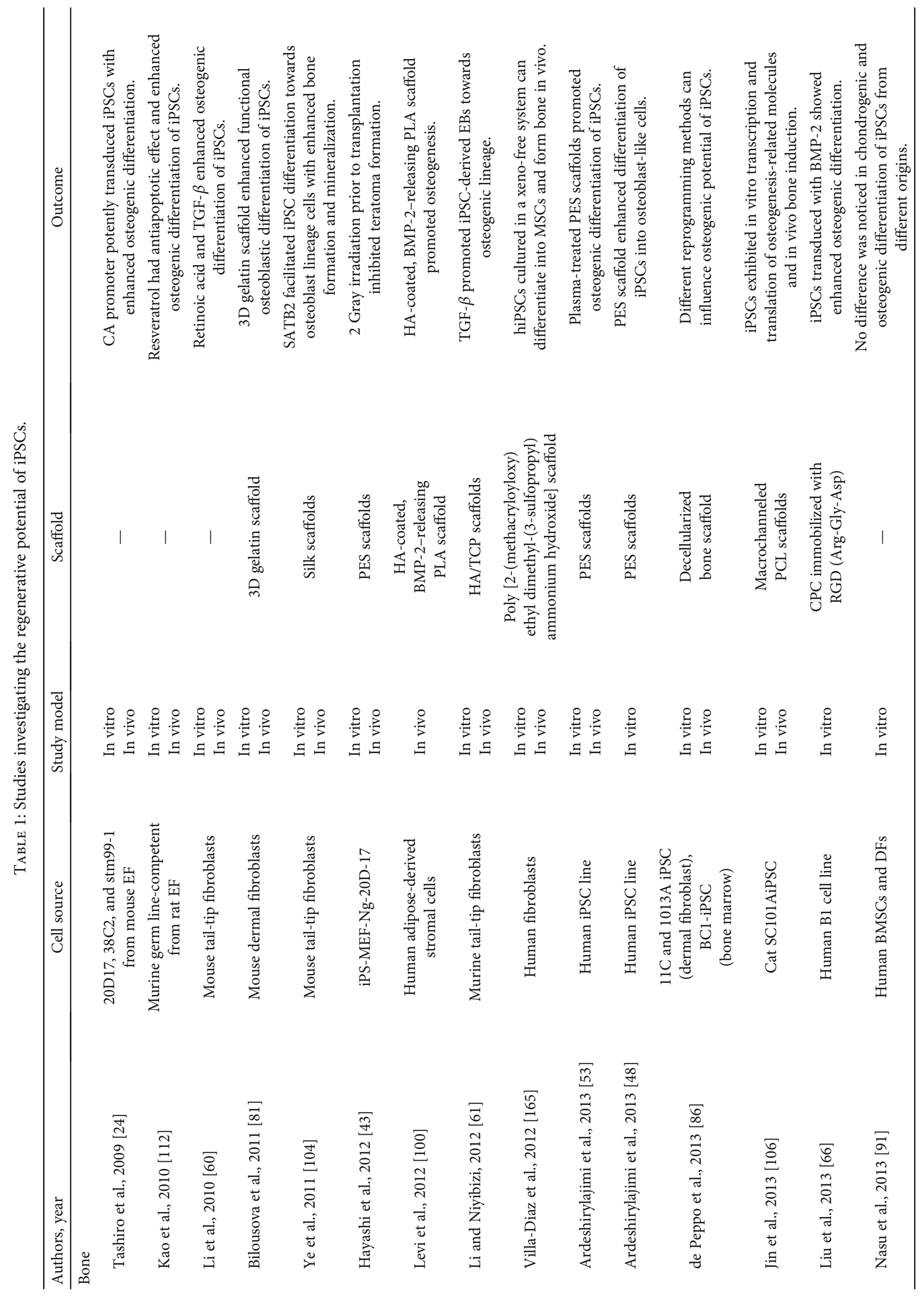




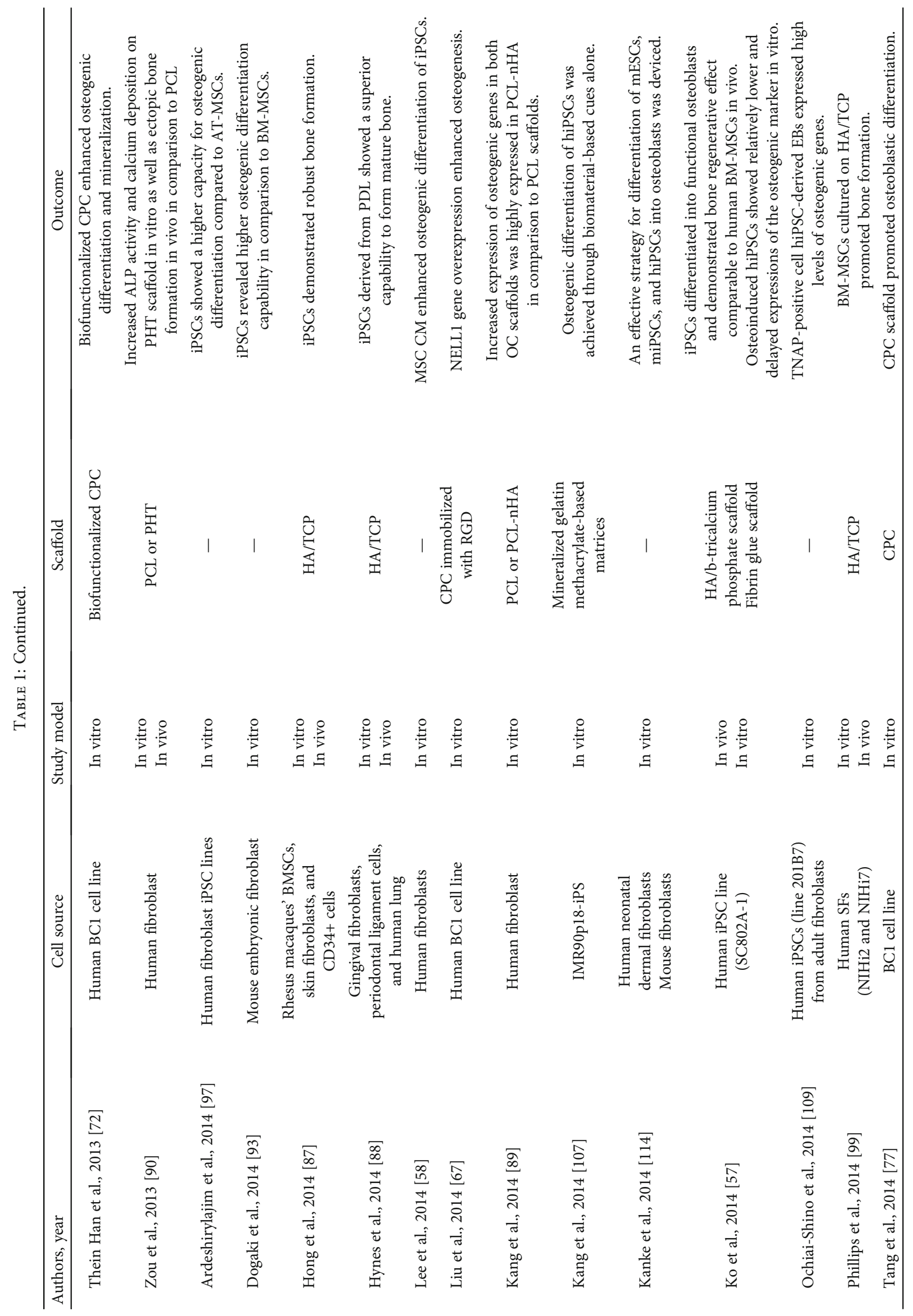




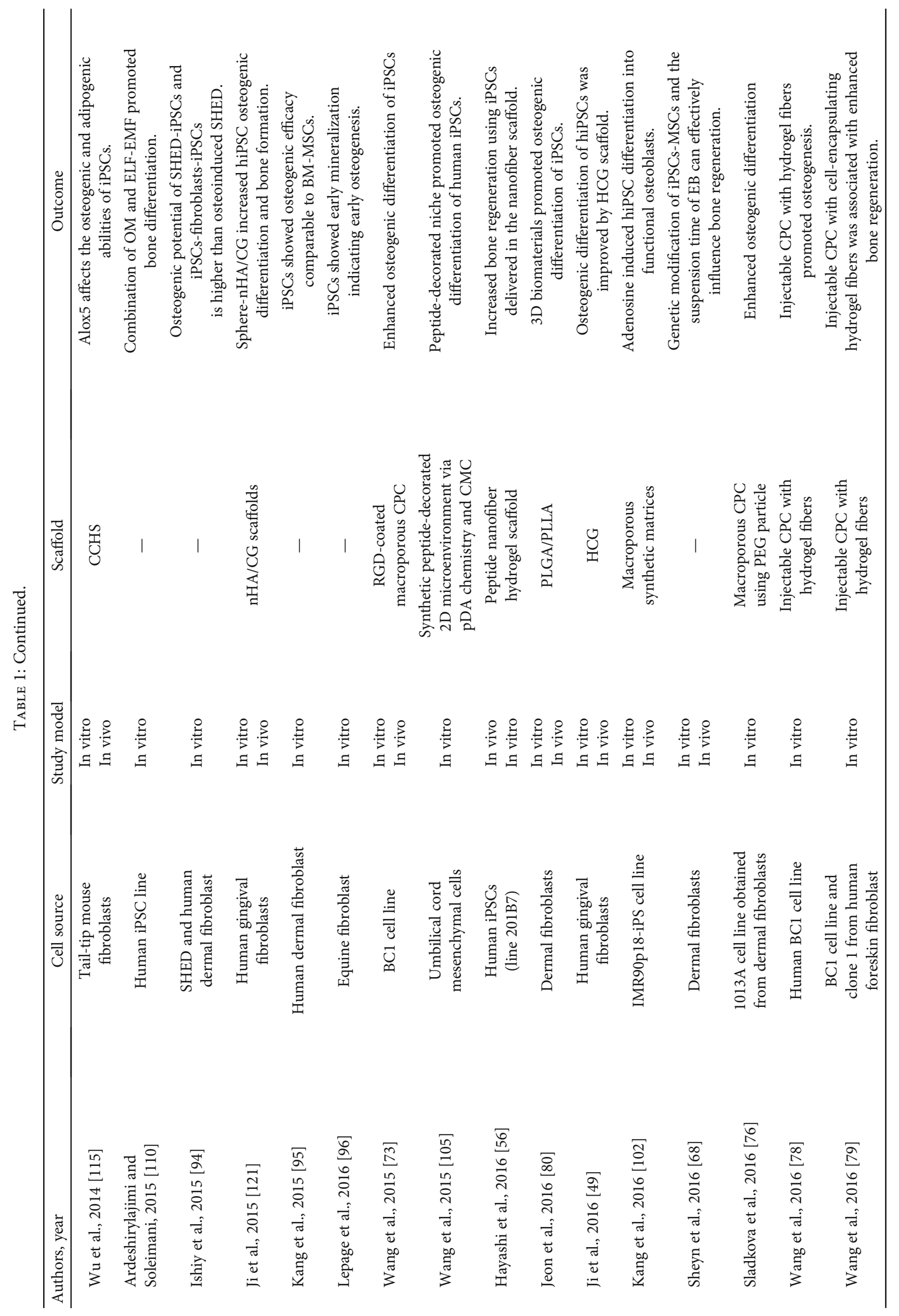




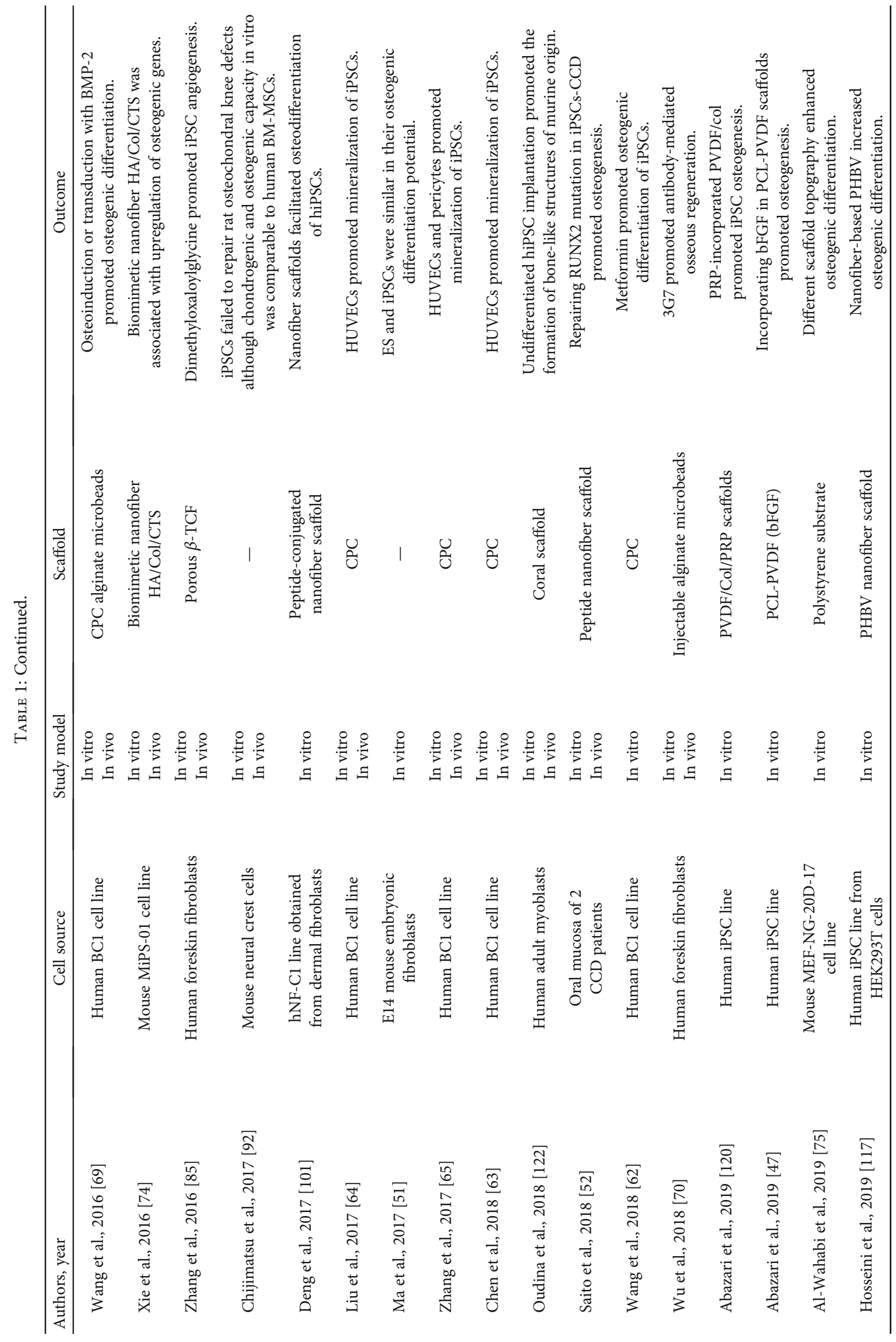




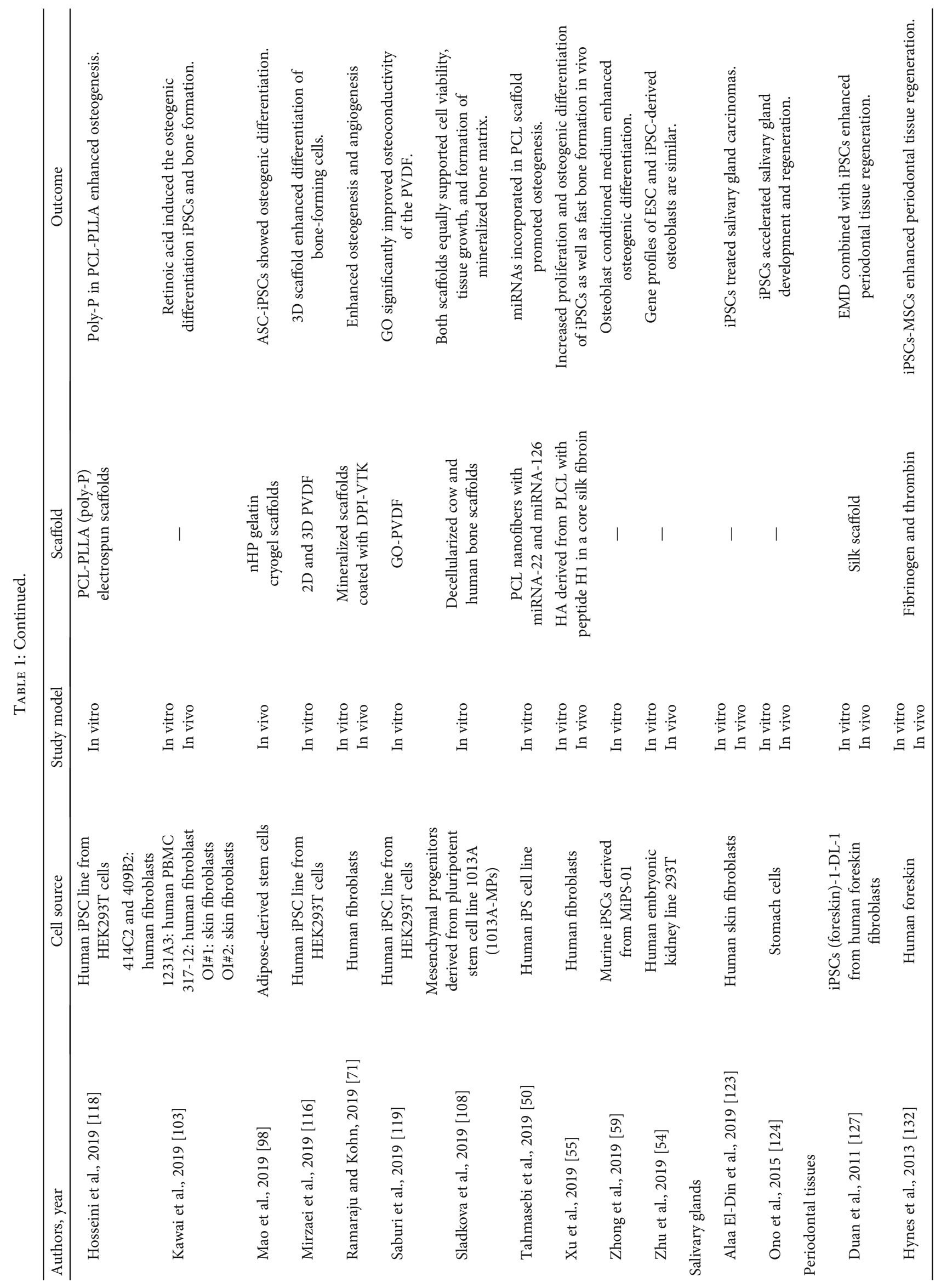




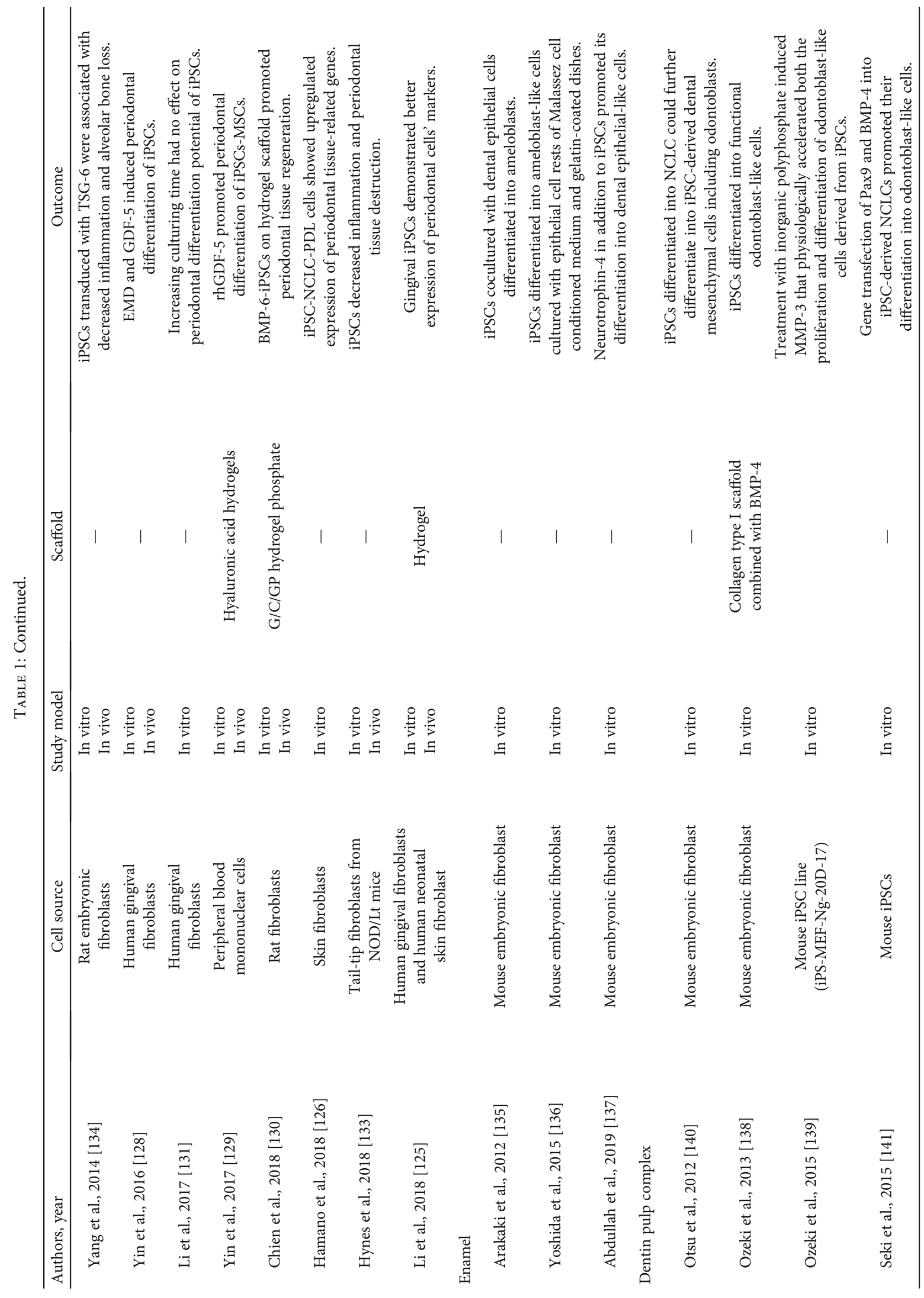




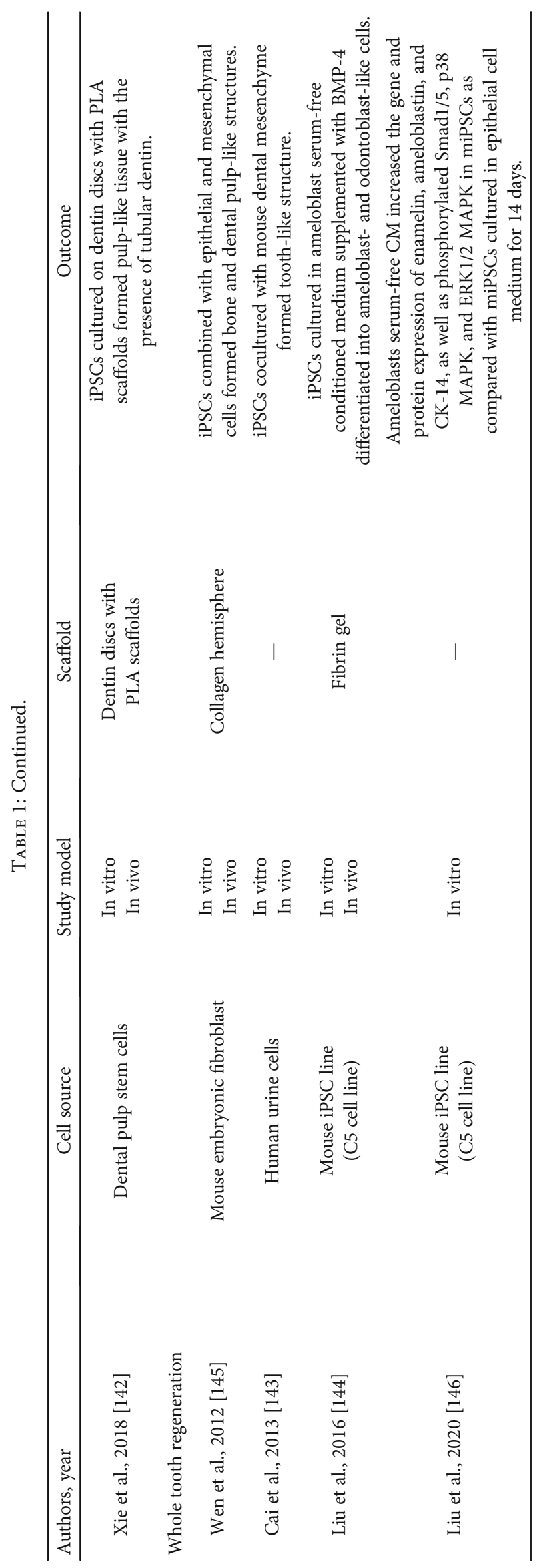


while opposite results were obtained upon injecting BMP-6early MSCs. It was concluded that iPSCs-MSCs obtained at early EB suspension time possessed a more pronounced stem cell phenotype and were capable of ectopic bone formation, whereas those cells obtained later acquired a more differentiated phenotype of osteoblasts and were capable of significant bone formation in vivo [68].

Similarly, genetic modification of human iPSCs-MSCs by either BMP-2 or NELL1 overexpression, followed by seeding of the modified cells on calcium phosphate cement (CPC) scaffold immobilized with RGD (Arg-Gly-Asp), showed significantly high expression of RUNX2, OCN, and COL1A1 $[66,67]$. Additionally, human iPSCs-MSCs that were either osteoinduced or transduced with BMP-2 demonstrated high expression levels of osteoblast-related genes [69]. Incorporating retinoic acid combined with TGF- $\beta 1$ or TGF- $\beta 1$ into murine iPSC-derived EB culture media enhanced mineralization and osteogenic differentiation [60, 61]. Additionally, human iPSCs-MSCs cultured in the presence of metformin and seeded on CPC scaffolds showed upregulated expression of osteoblast-related genes and proteins as well as increased mineralization. Induction of adenosine monophosphate(AMP-) activated protein kinase phosphorylation concomitant with increased RUNX2 expression was also evident [62]. Moreover, coseeding of human iPSCs-MSCs with human umbilical vein endothelial cells (HUVECs) on CPC scaffolds $[63,64]$ or coseeding with pericytes [65] enhanced osteogenesis and vascularization in vitro and in vivo with an upregulation expression of osteogenic (ALP, OCN, and COL1A1) and angiogenic genes (vascular endothelial growth factor (VEGF) and vascular endothelial cadherin).

Antibody-mediated osseous regeneration was recently described to impact in vivo bone regeneration. Human iPSCs-MSCs were combined with 3G7, an anti-BMP-2 antibody, that were hypothesized to facilitate the engagement of BMP-2 to their receptors on iPSCs-MSCs. 3G7 and iPSCs-MSCs were subsequently loaded on biocompatible, biodegradable alginate microbeads that were injected subcutaneously in rats. In vivo enhanced bone formation, mineralization, and vascularization associated with in vitro enhanced osteogenic differentiation were mediated through activation of the BMP-2/Smad1/RUNX2 pathway [70].

Biofunctionalization of the scaffold was further suggested to promote human iPSCs-MSCs osteogenic differentiation and vascularization, where human iPSCs-MSCs seeded on CPC scaffolds, treated with biofunctional agents and bioactive peptides [71-73] as well as murine iPSCs-MSCs seeded on biomimetic nanofibers of hydroxyapatite/collagen/chitosan (HA/COL/CTS), showed upregulation of RUNX2, OSX, ALP, and COL1A1 gene expression levels [74]. Furthermore, outgrowing cells from mouse iPSCs cultured on different polystyrene substrate topographies displayed upregulation of COL1A1 and RUNX2 [75]. Human iPSCs-MSCs seeded on microporous CPC scaffolds using polyethylene glycol (PEG) particles showed upregulation of RUNX2, COL1A1, ALP, OPN, and platelet-derived growth factor receptor-beta (PDGF-R- $\beta$ ) [76]. Similarly, human iPSCs-MSCs seeded on CPC $[62,77-79]$ or poly lactic-co-glycolic acid/poly L-lactic acid (PLGA/PLLA) scaffold combined with macrophages
[80] or fast degradable alginate microbeads [69] showed high expression of osteoblast-related genes. Moreover, murine iPSC-derived MSCs seeded onto three-dimensional gelatin scaffold revealed upregulation of several osteoblast-related genes in vitro and in vivo, following subcutaneous implantation in rats [81]. Demonstrating the key role of osteoprotegerin/receptor activator of nuclear factor $\kappa$ B ligand (OPG/RANKL) in orchestrating osteoblastic and osteoclastic action in bone remodeling, human iPSCs-MSCs were cocultured with iPSCs-macrophages committed to osteoblastogenesis and osteoclastogenesis, respectively, on HAbased PLGA/PLLA 3D scaffolds. Enhanced expression of bone-related genes upon monoculturing human iPSCsMSCs on HA-5 PLGA/PLLA was demonstrated as compared to HA-0 PLGA/PLLA. Coculturing induced upregulated expression of late osteogenic markers (OPN and OCN) and downregulated expression of early osteogenic markers (COL1A1, ALP, and RUNX2). Similar results were attained in vivo through implantation of HA-PLGA/PLLA scaffold loaded with human iPSCs-MSCs and iPSCs-macrophages subcutaneously in rodents [80].

\subsubsection{Osteogenic Potential of iPSCs-MSCs Obtained without} EB Formation. Another method proposed to obtain iPSCsMSCs relies on the dissociation of iPSC colonies, without prior formation of $\mathrm{EB}$, into a single cell suspension. The resulting cells are characterized as MSCs, either through flow cytometry or through cell passaging protocols, followed by osteogenic differentiation [82-84]. Dimethyloxaloylglycine (DMOG) promoted iPSCs-MSCs derived from human foreskin fibroblast angiogenesis in critical-sized calvarial rat defects [85]. DMOG enhanced the expression of angiogenic factors (hypoxia-inducible factor 1- $\alpha$ (HIF-1 $\alpha$ ) and VEGF) through PI3K/Akt intracellular pathway activation, with improved bone formation.

The osteogenic potential of iPSCs-MSCs in combination with different scaffolds was investigated in several studies [55, 86-90]. The subcutaneous implantation of osteoinduced episomal-iPSCs (generated using an episomal vector) derived from BM stromal cells and retro-iPSCs (generated using a retroviral vector) derived from DF cultured on decellularized bone scaffold in SCID mice for 12 weeks revealed high mineral content in the episomal-iPSCs as compared to retro-iPSCs [86]. On the other hand, retro-iPSCs displayed the formation of a uniform bonelike matrix with embedded cells, while episomal-iPSCs exhibited areas of dystrophic calcification [86]. The osteogenic potential of human fibroblast-derived iPSCs was evaluated in vitro and in vivo on synthetic polymer polycaprolactone (PCL) scaffold or PCL scaffold functionalized with natural polymer hyaluronan and ceramic tricalcium phosphate ceramic poly (3-hexylthiophene (TCP-PHT)) [90]. The osteoinduced iPSCs revealed a significant increase in ALP activity and calcium deposition on PHT scaffold in vitro as well as ectopic bone formation in vivo in comparison to PCL. Moreover, human fibroblast-derived iPSCs on PCL nanofibers alone or combined with nano-HA showed an increased expression of osteogenic genes (RUNX2, ALP, COL1A1, and OCN) in both scaffolds, even though they were 
expressed at a different time intervals, OCN was highly expressed in PCL-nano-HA in comparison to PCL scaffolds [89]. Similarly, the incorporation of short hydrophilic peptide $\mathrm{H} 1$ derived from connective tissue growth factor in a core silk fibroin (SF) combined with HA derived from poly (L-lactic acid-co- $\varepsilon$-caprolactone) (PLCL) resulted in increased proliferation and osteogenic differentiation of iPSCs-MSCs derived from human fibroblasts [55].

The interaction between HA/TCP ceramic particles and iPSCs-MSCs was subsequently investigated in vivo $[87,88]$. Rhesus macaques' iPSC-derived mesodermal stromal-like cells mixed with HA/TCP demonstrated robust bone formation when implanted subcutaneously for eight weeks [87]. Furthermore, the osteogenic potential of iPSCs-MSCs from gingival fibroblasts, periodontal ligament cells, and human lung combined with HA/TCP was compared following implantation in SCID mice subcutaneously [88]. Although the three types of iPSCs-MSCs were able to form mineralized tissue, iPSCs-MSCs derived from periodontal ligament cells showed superior capability to form mature bone and connective tissue, which led to a controversial assumption that even after induction, iPSCs may retain epigenetic memory of their origin [91]. The combination of HA derived from PLCL with osteoinductive peptide $\mathrm{H} 1$ in a core SF and iPSCs-MSCs derived from human fibroblasts resulted in faster bone formation in vivo as compared to SF/PLCL following eight weeks of implantation in calvarial mouse defects [55].

Yet, although most of the aforementioned studies highlighted the osteogenic potential of iPSCs-MSCs in bone regeneration, Chijimatsu et al. reported that MSCs derived from iPSCs-neural crest cells failed to repair rat osteochondral knee defects in vivo despite their demonstrated chondrogenic and osteogenic capacity comparable to human BMMSCs in vitro [92].

\subsubsection{Osteogenic Differentiation Capability of iPSCs} Compared to Other Types of Cells. The osteogenic differentiation ability of iPSCs-MSCs in comparison to MSCs was examined in a variety of studies [86, 93-95]. A study on iPSCs showed a delayed expression of osteogenic markers such as COL1A1 and bone sialoprotein (BSP) as well as weaker osteoblastic differentiation and mineral deposition, compared to human BM-MSCs in vitro [57]. Human fibroblast-derived iPSCs reprogrammed by mRNA (mRNA-iPSCs) or polycistronic lentiviral vector (lenti-iPSCs) were compared to BMMSCs [95]. Both methods of transduction produced cells that were similar in their morphology and surface antigen to BMMSCs. lenti-iPSCs revealed faster and more homogeneous calcium staining than mRNA-iPSCs. Although the expression of RUNX2, ALP, and OCN was stronger in BM-MSCs as compared to iPSCs-MSCs, the opposite was demonstrated for COL1A1 expression. Both iPSCs-MSCs showed osteogenic efficacy comparable to BM-MSCs. Similarly, osteoinduced mouse iPSCs-MSCs revealed the same surface antigen profile and higher osteogenic differentiation as BM-MSCs [93]. ALP, OSX, RUNX2, and OCN were intensely upregulated in osteoinduced iPSCs-MSCs aside from the formation of a mineralized matrix at day 14 of osteogenic induction. retro-iPSCs and episomal-iPSCs exhibited higher ALP gene expression than human ES cells [86]. Moreover, the osteogenic potential of iPSCs-MSCs derived from either human deciduous teeth or human DF was higher than that of osteoinduced SHED [94]. iPSCs-MSCs derived from equine fibroblast iPSCs were compared to MSCs derived from newborn foals' umbilical cord blood (CB-MSCs) [96]. Von Kossa and alizarin red staining of iPSCs-MSCs showed early mineralization indicating early osteogenesis which was consistent with the results obtained from CB-MSCs.

Similarly, Ardeshirylajimi et al. [97] compared the biological behavior and osteogenic differentiation potential of human iPSCs and adipose tissue (AT-MSCs). iPSCs confirmed high osteogenic differentiation potential and superior ALP activity and mineralization level. Notably, AT-MSCs expressed greater levels of RUNX2, while iPSCs expressed higher levels of OCN and osteonectin during differentiation which may be a result of their increased proliferation rate compared to AT-MSCs [97]. In vivo comparison of osteogenic potentials between adipose-derived stem cells (ASCs) and ASC-iPSCs loaded on nano-HA gelatin cryogel scaffolds revealed a superior osteogenic differentiation with enhanced osteogenic marker expression of COL1A1 and RUNX2 in the ASC-iPSCs group, proposing ASC-iPSCs as an alternative cell source in bone tissue engineering with a good differentiation ability [98].

On the other hand, the osteogenic potential of iPSCs derived from human skin fibroblasts was compared to iPSCs derived from BM-MSCs cultured on HA/TCP implanted subcutaneously in nude mice [99]. No differences in bone formation were revealed between iPSCs from different origins. In addition, the bone regeneration ability of adiposederived stromal cells- (AS-) iPSCs was compared to human ES cells cultured on HA-coated PLGA scaffold with or without releasing BMP-2 in calvarial mouse defects [100]. Greater bone regeneration as well as upregulation of osteogenic markers was found in both AS-iPSCs and ES cells loaded on HA-PLGA releasing BMP-2 as compared to nonreleasing BMP-2 [100].

4.1.4. Factors to Improve the Osteogenic Potential of iPSCs (Figure 2). Exploring the therapeutic potential of iPSCsMSCs in dental and nondental tissue regeneration entails the optimization of the factors that would enhance their osteogenic potential for future clinical applications. Genes, isozymes, laser application, suspension time of EBs, transduction method, natural antioxidant and anticancer products, and constituents of the scaffold material are factors that could enhance or affect the osteogenic potential of iPSCs. In order to attain iPSC osteogenic commitment, various inductive factors were applied including chemical inducers, biomolecules [101-103], growth factors [100], gene modification [104], two-dimensional culture environment [105], and modified three-dimensional scaffolds [100, 101, 106-108]. Tissue-nonspecific alkaline phosphatase (TNAP) was demonstrated to influence the osteogenic differentiation potential of iPSCs, where TNAP-positive cells isolated from human EBs derived from iPSCs and cultured in osteogenic media expressed high levels of OSX, RUNX2, COL1A1, 


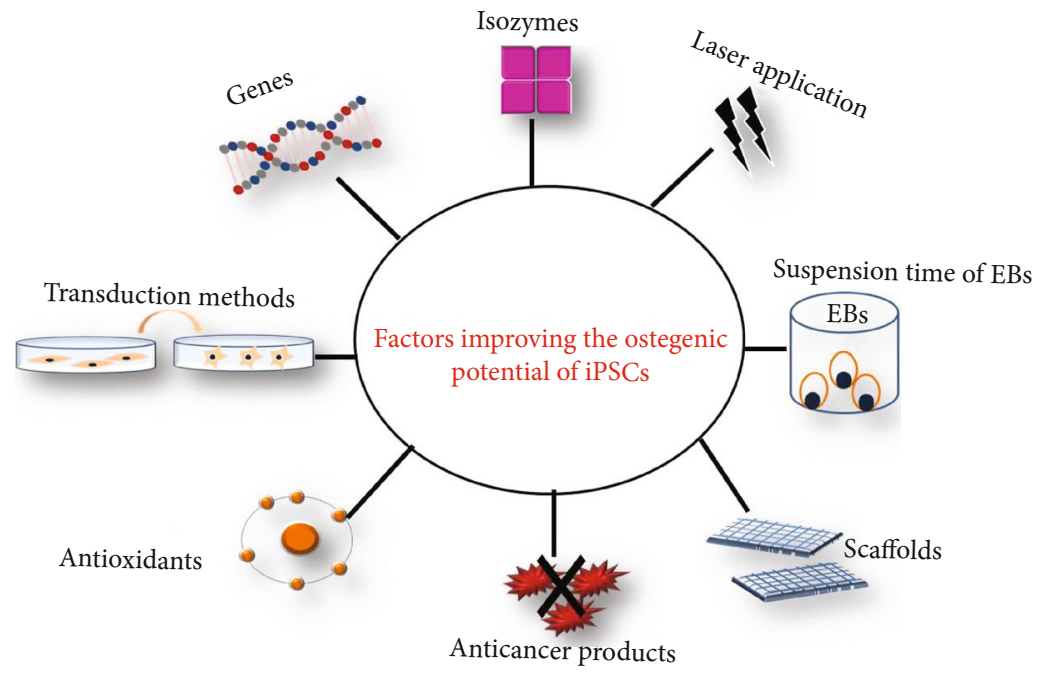

FIGURE 2: Diagram summarizing factors which may affect osteogenic potential of iPSCs.

BSP, and OCN as well as generated mineralized nodules and revealed a significant expression of osteocyte marker genes, including sclerostin, neuropeptide Y, and reelin [109]. Similarly, extremely low-frequency electromagnetic field (ELFEMF) $(50 \mathrm{~Hz}$ and $1.5 \mathrm{mT})$ also significantly improved the osteogenic potential of iPSCs [110]. Resveratrol a natural polyphenol found largely in red grapes, nuts, pomegranates, and red wine [111] was also found to facilitate osteogenic differentiation of iPSCs, with increased osteogenic gene expression and mineralization content [112]. Growth factors such as recombinant human- (rh-) BMP-2 have been shown to positively modulate osteogenic transformation of iPSCs. Adding rh-BMP-2 to the osteogenic media improved the osteogenic potential of iPSCs derived from human AS through significant upregulation of osteogenic markers RUNX2 and OCN [100]. In vitro results showed that $3 \mathrm{wt} / \mathrm{vol} \%$ nano-HA in chitosan/gelatin (CG) and miRNAs increased the expression of osteogenic-related genes [49, 50], formed bone-like tissue in vivo [49], and upregulated the OCN and OPN protein expression on day 21 after culturing [50].

Even though growth factors can endorse the osteogenic differentiation of iPSCs, their effects are limited due to their short half-lives and uncontrolled degradation. In contrast, gene modification of iPSC-derived cells can attain a longterm effect via retaining a relatively stable local concentration of these factors [113]. Certain genes such as nuclear matrix protein SATB2 have been transduced into iPSCs to promote osteodifferentiation [104]. An efficacious strategy for differentiating human iPSCs into osteoblasts involves using four small molecules including CHIR99021 (CHIR), cyclopamine (Cyc), smoothened agonist (SAG), and helioxanthinderivative 4-(4-methoxyphenyl) pyrido $\left[4^{\prime}, 3^{\prime}: 4,5\right]$ thieno [2,3-b] pyridine-2-carboxamide (TH) under chemically well-defined conditions [114]. Ex vivo gene therapy of SATB2-modified iPSCs increased the levels of calcium nodule formation, ALP activity, and osteogenic genes in vitro. Subsequent implantation of the transduced cells on silk scaf- fold encouraged bone regeneration in critical-sized calvarial defects [104]. On the contrary, iPSCs derived from tail-tip fibroblasts of Alox 5 knockout mouse demonstrated significant downregulation of early and late osteogenic gene levels with significant upregulation of adipogenic markers. Still, loading Alox5-KO-iPSCs on collagen/chitosan/hydroxyapatite scaffolds induced significantly less new bone formation in rat cranial critical-sized defects as compared to wildiPSCs [115].

Interestingly, iPSC origin demonstrated no effect on iPSC osteogenic potential. The osteogenic differentiation properties of human iPSCs derived from BM-MSCs and DFs demonstrated no marked differences in gene expression profiles as well as in the methylation profile. Moreover, the chondrogenic and osteogenic differentiation properties of iPSCs from different cells' origin showed no significant differences, although a higher tendency was reported in DF-derived iPSCs [91]. Yet, different reprogramming methods could affect the osteogenic differentiation of iPSCs [86]. iPSCs derived from DF reprogrammed by retroviral vectors (retro-iPSCs) or Sendai virus (Sendai-iPSCs) cultured on decellularized bone scaffold in perfusion bioreactors demonstrated a new bone-like matrix with the highest cell density in Sendai-iPSCs, while retro-iPSCs showed poor osteogenic differentiation [86].

Human iPSCs derived from human embryonic kidneyEB were utilized to compare the osteoinductive properties of 3D nanofibrous scaffold of polyvinylidene fluoride (PVDF) with 2D scaffold [116] as well as to assess electrospun poly (3-hydroxybutyrate-co-3-hydroxyvalerate) (PHBV) nanofiber scaffold [117]. iPSCs revealed significantly high ALP activity, calcium content, and osteogenicrelated genes after seeding on 3D PVDF [116] and PHBV scaffolds [117]. Moreover, OCN and OPN protein expressions were elevated on day 21 after cell seeding [116, 117]. Utilizing different ratios from nano-HA [49] or different miRNAs (miR-22 and miR-126) [50] in chitosan/gelatin (CG) scaffold or electrospun PCL nanofiber 
scaffold, respectively, was also reported to affect the osteogenic differentiation of human iPSCs. Furthermore, incorporating basic fibroblast growth factor (bFGF) in PCL-PVDF scaffold [47] or polyphosphate (poly-P) in PCL/PLLA electrospun scaffolds [118] or graphene oxide (GO) in PVDF nanofibers [119] or platelet-rich plasma in PVDF/collagen nanofibrous scaffolds [120] significantly increased the survival rate of iPSCs and upregulated ALP activity, mineralization content, and expression of preosteoblast- and osteoblast-related genes in iPSCs loaded on PCL-PVDF (bFGF), PCL-PLLA (poly-P), PVDF-GO, or PVDF/Col/PRP scaffolds [47, 118-120]. A xeno-free nanofiber scaffold conjugated with vitronectin peptide upheld pluripotency and proliferation of seeded human iPSCs. Interestingly, this osteogenic culture system promoted direct osteodifferentiation of human iPSCs, as confirmed by the cellular morphology, ALP assay, and RT-PCR analysis combined with immunofluorescence results [101]. A recent report confirmed the osteogenic differentiation of human iPSCs into osteoblast-like cells with enhanced calcified nodule formation under the influence of retinoic acid in vitro and membranous bone tissue formation in vivo without scaffolds [103]. Under osteogenic conditions, human iPSCs cultured on PCL scaffolds confirmed osteogenesis by OPN detection using quantitative PCR and by western blotting. Further subcutaneous implantation in mice revealed marked calcium deposition and positive OCN immunostaining, with no signs of teratoma formation, following the osteogenic induction of human iPSCs [106]. The osteogenic potential of human iPSC-derived mesodermal progenitor cells (hiPSC-MP) on decellularized tissue matrices as scaffolding materials and human bone scaffolds in osteogenic medium under dynamic conditions was compared in perfusion bioreactors. Both scaffolds equally promoted cell viability and mineralized tissue formation [108]. Peptide-decorated 2D culture microenvironment developed through polydopamine (pDA) chemistry with subsequent carboxymethyl chitosan successfully promoted osteogenic differentiation of human iPSCs in vitro [105]. These results were supported by enhanced ALP activity, gene expression, and corresponding protein expression as well as the amount of calcium deposition [105]. Human iPSCs isolated from clinically discarded human gingival tissues were seeded on both sphere-shaped or rod-shaped nano-HA/CG scaffolds. Notably, the sphere-shaped nano-HA in HA/CG scaffolds greatly improved the osteogenic differentiation of human iPSCs as compared to rod-shaped. Consequently, human iPSCs and sphere-shaped nano-HA/CG composites generated a significant amount of bone in vivo [121].

Adenosine-induced differentiation of human iPSCs (AdiPSCs) loaded on poly (ethylene glycol) diacrylate-coacryloyl 6-aminocaproic acid (PEGDA-co-A6ACA) macroporous hydrogel into functioning osteoblast, in growth medium lacking any other osteoinductive factors, revealed progressive dense bone tissue formation. Furthermore, AdiPSCs implanted in critical-sized cranial bone defects in mice showed uniform hard tissue formation all over the cranial defect that was integrated with the adjacent bone without teratoma formation [102]. Moreover, ex vivo two-dimensional and three-dimensional cultures and mineralized gelatin methacrylate- (GelMA-) based matrices containing CaP mineral endorse the osteogenic differentiation of human iPSCs in osteoinductive factors free growth medium via the dissociation of $\mathrm{Ca}^{2+}$ and $\mathrm{PO}_{4}{ }^{3-}$ ions in a permissive environment through various signaling pathways [107]. Similarly, ectopically implanted human iPSCs seeded on coral scaffolds in mice demonstrated the expression of bone-like structures through the release of osteoinductive factors including BMPs [122]. Paradoxically, the rapid disappearance of human iPSCs due to early cell death was associated with an increase in the osteogenic genes. To settle these conflicting trends, the authors investigated the paracrine effect of bioactive CM from human iPSCs. Interestingly, human iPSC CM promoted the osteogenic differentiation of human MSC osteogenic differentiation as well as upregulated the expression of BMP-2, BMP-4, and BMP-6 genes and enhanced extracellular matrix mineralization [122].

4.2. iPSCs and Salivary Gland Regeneration. iPSC therapeutic and regenerative potentials were exploited in the treatment of salivary glands' diseases. In an in vivo study, iPSCs were utilized for treating salivary gland carcinoma induced in mice. Although iPSCs improved salivary gland function detected by a significant increase in the gene expression of $\alpha$-amylase, the glands retained some malignant architecture including minor acinar, ductal, and vascular degenerative changes [123].

In an attempt to uncover the paracrine role of iPSCs in salivary gland regeneration, embryonic submandibular gland (SG) cells and mouse green fluorescent protein iPSCs (iSG) were cocultured. More developed epithelial structures were evident upon coculturing than in monoculture of embryonic SG cells. Upon morphological analysis of the regenerated tissues, iSG had a greater number of small acinar-like structures than that in SG cells. Additionally, analysis of differentiation markers among groups showed lower Sox2, c-Myc, and Nanog gene expression and higher Klf4 and Aqp5 gene expression in iSG with a remarkable regenerative capacity [124].

4.3. iPSCs and Periodontal Tissue Regeneration. iPSC differentiation into periodontal regenerative cells is affected by a variety of factors including cell source [125], culturing media [126], coculturing with inducing factors such as enamel matrix derivative (EMD) [127, 128], recombinant growth/differentiation factor-5 (GDF-5) [128, 129] or BMP-6 [130], the number of cellular passages [131], and type of scaffold used [130]. EBs generated from human gingival fibroblast and human neonatal skin fibroblast-derived iPSCs were induced into periodontal progenitor cells, which were then implanted on hydrogel scaffold subcutaneously in SCID rats. Owing to the cells' inherent epigenetic memory, iPSCs derived from gingival fibroblasts showed a higher expression of periodontal cell markers in vitro, including BSP, cementum protein 1 and periostin, and a formation of mineralized structure in vivo, with no teratoma formation observed with either cell types [125]. Neural crest cells derived from human skin fibroblast iPSCs cultured in combination with PDL cells' extracellular matrix showed a higher proliferation rate and a stronger expression of periodontal cell markers, including COL1A1, fibrillin-1, OPG, and periostin, as compared to 
cells cultured with either fibronectin, laminin, or dermal fibroblast extracellular matrix [126].

Culturing EB derived from human foreskin iPSCs in combination with EMD gel promoted the expression of RUNX2, an early osteogenic marker, but inhibited the expression of OCN, a late osteogenic marker, and mineralization in vitro. To assess the effect of iPSCs and EMD on osteogenic differentiation and periodontal regeneration in vivo, EBs derived from mouse iPSCs were seeded on apatitecoated silk fibroin scaffolds with EMD before implantation in the periodontal fenestration defect rat model. Following iPSCs-EMD in vivo transplantation, OCN, RUNX2, and OSX expression was higher than those in the control group which was attributed to the ability of EMD to recruit a large number of osteogenic cells. Moreover, iPSCs-EMD were able to induce the formation of new bone almost filling the periodontal defect, promoted the formation of new cementum covering the surface of the root, and stimulated the formation of periodontal fibers perpendicular to the root surface proving that iPSCs-EMD can further be an efficient tool in periodontal regeneration [127].

The periodontal differentiation potential of iPSCs derived from human gingival fibroblasts and treated with growth differentiation factor- (GDF-) 5 was investigated at different passages $[5,10,15,20]$. All iPSCs-GDF-5-treated passages revealed a high proliferative ability and attained fibroblast-like cell morphology, significant production of calcified nodules, and upregulated expression of bone-related gene (OCN and BSP), periodontal ligament-related gene (periostin and vimentin), and cementum-related genes (cementum attachment protein and cementum protein 1) as compared to their untreated controls [131]. Yet, the periodontal differentiation capability of iPSC-derived MSCs, obtained either from human gingival tissues or from peripheral blood mononuclear cells, was significantly increased after their treatment with recombinant human GDF-5 (rhGDF-5) $[128,129]$. This was confirmed by the marked expression of periodontal tissue-related genes $(\mathrm{OCN}$, periostin, and cementum attachment protein). On the contrary, BM-MSCs treated with rhGDF-5 demonstrated an insignificant expression of periostin and CAP, despite the high expression of OCN. Similar results were attained upon loading PKH67-labeled iPSCs-MSCs-rhGDF-5 on hyaluronic acid and subsequent implantation into the dorsal surface of 6-8-week-old male athymic nude mice. Moreover, after 4 weeks of culture with rhGDF-5, both BMSCs and iPSCsMSCs showed noticeable mineralization with nodule formation [129]. Chitosan/gelatin/glycerol phosphate hydrogel 3D scaffold seeded with osteogenic-induced rat fibroblastderived iPSCs and BMP-6 applied to periodontal defect created on the root surface of the maxillary first molar in rats significantly downregulated inflammatory cytokines interleukin 8 (IL-8), tumor necrosis factor alpha (TNF- $\alpha$ ), and IL- $1 \beta$ and promoted bone and periodontal tissue regeneration [130]. Additionally, human foreskin iPSC-derived MSCs, clotted with fibrinogen and thrombin implanted in periodontal fenestration defect in SCID rats, also revealed a significant increase in newly formed mineralized tissue area percentage [132].
Mesenchymal stromal cells derived from tail-tip fibroblast iPSCs (iPSCs-MCs) revealed immunomodulatory capabilities of the periodontal inflammatory destruction, which may offer a potential therapeutic modality for periodontal disease. In this context, a bacterial-induced periodontitis mouse model was established through local application of Porphyromonas gingivalis into the oral cavity and its systemic administration, while an acute inflammation model was created via subcutaneous implantation of heat-killed Porphyromonas gingivalis-impregnated sponge in rats. Rats were treated by systemic injection of iPSCs-MCs into the tail vein seven days following periodontitis establishment or by local iPSCs-MCs administration into the implantation site. iPSCs-MCs showed a significant reduction in inflammation and alveolar bone loss in the periodontitis rats' model. Moreover, local or systemic iPSC treatment in the acute inflammation model showed a reduced expression of the proinflammatory cytokine CXCL1, while local iPSCs-MCs administration resulted in a significant reduction in the inflammatory score [133]. Similarly, periodontitis was induced around the maxillary first molar bilaterally in female rats by ligature and subsequent infection with Porphyromonas gingivalis. The rats were treated intravenously and topically with rat iPSCs-MSCs reprogrammed from rat embryonic fibroblasts and transduced with tumor necrosis factor alpha-stimulated gene-6 (TSG-6) (iPSCs-MSCs/TSG-6). A significant downregulated level of alveolar bone loss, a few number of TRAPpositive osteoclasts, and serum interleukin $1 \beta$ (IL-1 $\beta$ ) and tumor necrosis factor alpha (TNF- $\alpha$ ) were demonstrated as compared to untreated rats [134].

4.4. iPSCs and Enamel Regeneration. Ameloblasts are crucial cell populations required for enamel formation. The ability of mouse iPSCs (miPSCs) to differentiate into ameloblast was investigated [135], where miPSCs cocultured with dental epithelial cells differentiated into ameloblasts, exhibiting epithelial cell-like morphology in addition to expressing ameloblastic markers (ameloblastin and enamelin) and epithelial markers (p63 and cytokeratin- (CK-)14), suggesting an epithelial-mesenchymal interaction role in tooth development. Similarly, miPSCs differentiated into ameloblast-like cells under feeder-free conditions, using cultured epithelial rests of Malassez (ERM) cell CM and gelatin-coated dishes [136]. The differentiated ameloblastlike cells demonstrated an increase in expression of CK14, amelogenin, and ameloblastin in comparison to miPSCs cocultured with ERM cells. The levels of amelogenin expression in ameloblast-like cells were significantly higher than those in miPSCs cocultured with ERM cells throughout the experiment, while ameloblastin increased significantly on day 14. Moreover, the addition of neurotrophin- 4 to miPSCs under serum-free culture conditions during $\mathrm{EB}$ formation leads to their differentiation into dental epithelial-like cells with the upregulation of epithelial and ameloblastic markers [137]. These studies highlighted the potential differentiation ability of iPSCs into ameloblasts confirming that iPSCs could be a new cell source for enamel regeneration. 
4.5. iPSCs and Dentin Pulp Complex Regeneration. The generation of odontoblast cells from iPSCs could open new opportunities for treating dentinal and/or pulpal damage. Epithelial-mesenchymal interactions are required for differentiating iPSCs into odontoblasts. Herein, the study described the "hanging drop" technique for differentiating miPSCs into odontoblast-like cells exploiting such an interaction. iPSCs were differentiated into EBs and then cultured on a collagen scaffold (CS) in combination with BMP-4 (CS/BMP-4). The generated cells intensely expressed mature odontoblast markers, dentin sialoprotein (DSP), and dentin matrix protein-1 (DMP-1) and presented physiological as well as functional features of odontoblasts [138]. Moreover, in an in vitro model, matrix metalloproteinase- (MMP-) 3 small interfering RNA was transfected into odontoblast-like cells derived from iPSCs. Strikingly, treatment with inorganic polyphosphate induced MMP-3 that physiologically accelerated both proliferation and differentiation of odontoblastlike cells, thereby hypothesized to provide some protection to the cells against the detrimental effects of inflammation and pulp capping materials. Additionally, DSPP and DMP1 mRNA expressions were upregulated [139].

Under modified culture protocols, miPSCs were differentiated into neural crest-like cells (NCLCs) that could further differentiate into iPSC-derived dental mesenchymal cells (DMC) including odontoblast progenitor cells. Results showed that iPSC-derived NCLC expressed NC markers as demonstrated by immunocytochemistry, flow cytometry, and RT-PCR. Furthermore, NCLC expressed MSC markers, in addition to Pax9 and DSP, proving their capacity to differentiate into dental mesenchyme, when cultured with dental epithelium [140]. Interestingly, gene transfection of Pax9 and BMP-4 into iPSC-derived NCLCs promoted their differentiation into odontoblast-like cells, thus prompting signaling modulation of DMP-1 and DSPP expression, associated with odontoblastic differentiation of miPSCs [141]. In another study, dental pulp stem cells (DPSCs) were reprogrammed into iPSCs; then, the cells were seeded on dentin discs with PLLA scaffolds and implanted subcutaneously in mice. Amazingly, iPSCs generated a pulp-like tissue having tubular dentin, while in vitro, iPSCs maintained the odontogenic and mineralization potential after long-term expansion opposite to DPSCs [142].

4.6. iPSCs and Whole Tooth Regeneration. In addition to ameloblastic and odontoblastic differentiation potential of iPSCs, the capability of iPSCs in whole tooth regeneration was investigated [143-145]. miPSCs which clearly express odontogenic and osteogenic genes following their induction were implanted combined with epithelial and mesenchymal cells in a tooth germ model and transplanted into subrenal mouse capsule [145]. After four weeks of implantation, the formation of bone, dental pulp-like, and irregular tooth-like structures was demonstrated. Additionally, OPN was expressed in the apical region of the tooth-like structure. Notably, implantation of miPSCs alone failed to form dental or bone-like structures in contrast to its combined implantation with epithelial and mesenchymal cells.
Human iPSCs, derived from urine cells, were differentiated into epithelial sheets and cocultured with mouse dental mesenchyme, demonstrating an ability to form tooth-like structures such as enamel organ, enamel space, dentin, and dental pulp with physical and chemical properties similar to human teeth [143]. Further, through specific human antigen expression, it was revealed that iPSC epithelial sheets differentiated into ameloblast, while dental mesenchymal cells gave rise to the rest of the formed dental tissues. Interestingly, mouse dental mesenchymal cells alone formed bone-like tissue rather than tooth-like structure. Furthermore, miPSCs cultured in ameloblast serum-free CM supplemented with BMP-4 displayed the ability to form ameloblast- and odontoblast-like cells [144]. In addition, ameloblast serumfree CM increased the gene and protein expression of enamelin, ameloblastin, and CK-14, as well as phosphorylated Smad1/5, p38 MAPK, and ERK1/2 MAPK in miPSCs as compared with miPSCs cultured in epithelial cell medium for 14 days. Smad1/5 signaling transduction regulates the ameloblastic differentiation of miPSCs induced by ameloblast serum-free CM as the inhibition of Smad1/5 phosphorylation significantly reversed the increased the previously mentioned expression profile [146]. These results raise the possibility of iPSCs' use in whole tooth engineering opening a new gateway for biological tooth replacement.

\section{Challenges Facing iPSCs' Human Clinical Applications}

One of the major drawbacks that could hinder iPSCs' clinical application is their reported chromosomal instability and the underlying risk of tumor formation, which constitutes a substantial health hazard $[12,147]$. Undifferentiated iPSCs' pluripotency and their ability to differentiate into tissues derived from the three germ layers are an incentive to teratoma formation, which is used as an assay to test their pluripotency $[33,148]$. Moreover, iPSCs express several oncogenic genes [149]. Owing to iPSCs' unique properties, the generated tumor properties and origin are highly unpredictable and vary with the transplanted cell number as well as the utilized cell line [150]. In addition to their innate tendency for teratoma formation, the method of gene transduction can also increase the risk of tumorigenesis particularly due to the use of viruses that integrate their genome into the reprogrammed cells, as previously discussed. Currently, several attempts are carried out to overcome this through the use of nonviral vectors $[25,26]$ but are hindered by their lower transfection efficacy, especially following iPSC passaging.

Luckily, utilizing terminally differentiated iPSCs prior to implantation in addition to using nonviral vectors can help reduce risk of tumor formation [151]. Moreover, iPSCs can be reprogrammed via Oct3/4, Sox2, and Klf4, while omitting c-Myc which is a potent oncogene $[14,15,152]$. However, even following iPSC terminal differentiation, some cells may escape differentiation. Residual undifferentiated or partially differentiated iPSCs in the cellular transplants may cause teratoma formation upon implantation in the recipient tissues [153, 154]. Furthermore, iPSCs could retain epigenetic memory, which may affect their subsequent 
differentiation and direct them into lineages related to their parent cells $[155,156]$.

Another limitation associated with most current stem/progenitor cell isolation and expansion protocols lies in the utilization of xenogeneic-derived products in iPSC protocols. iPSCs are usually cultured on xenogeneic feeder cells that maintain the cells in an undifferentiated state without affecting their pluripotency [157], as well as fetal bovine serum that represents an important culture medium constituent [151, 158]. Using xenogeneic products in clinical trials could elicit an immunogenic reaction, carry a risk of disease transmission $[151,158]$, and affect reproducibility, as the exact composition of bovine serum varies greatly [159]. An additional problem creating an obstacle for the clinical application of iPSCs is the reduced generation efficacy [23], where iPSC generation efficiency using fibroblasts is extremely low. Even though generation efficiency is 4 to 10 times greater using dental pulp stem cells than fibroblasts, it is still relatively low for application in regenerative medicine [151].

\section{Short- and Long-Term Perspectives of iPSC- Mediated Tissue Regeneration}

Despite that iPSCs have shown promising results in regenerative medicine, a number of issues are yet to be resolved to allow their translation into clinical application while minimizing their potential side effects. Coculturing iPSCs with cells and growth factors could provide a promising solution to overcome tissue engineering challenges through mimicking in vivo conditions to optimize tissue regeneration results. Upon coculturing iPSCs-MSCs with iPSCs-macrophages committed to osteoblastogenesis and osteoclastogenesis, an OPG/RANKL milieu could be provided [80]. Similarly, coculturing iPSCs with dental epithelial and mesenchymal cells can reproduce epithelial-mesenchymal interaction signals orchestrating the process of tooth development. So far, securing an epithelial-mesenchymal interaction represents a great obstacle in whole tooth regeneration [135, 143]. Epithelial-mesenchymal interaction signals thereby remain to be the key towards inducing the differentiation of iPSCs into ameloblasts and other dental cells, which is the first step in whole tooth regeneration. Moreover, defining the best combination of iPSCs, signaling molecules such as growth factors, and scaffold biomaterials and determining the ideal architectural design of the scaffold $2 \mathrm{D}$ or $3 \mathrm{D}$, sphere- or rod-shaped, remain crucial for various applications of iPSCs in dental and paradental tissue regeneration.

Transduction of repaired, edited, and/or modified genes in iPSCs could be a beneficial tool for treating various disorders. In this context, repairing RUNX2 gene mutation in iPSCs derived from cleidocranial dysostosis patients [52] as well as transducing nuclear matrix protein SATB2 [104] and Alox 5 gene into iPSCs promoted osteodifferentiation [115]. Besides, Pax9 and BMP-4 gene transfection into iPSC-derived NCLCs promoted odontoblast-like cell differentiation [141] and attained a long-term effect of these factors rather than the short-term effect acquired following their local application [113].
iPSCs' extracellular vesicles, containing protein, mRNA, and miRNA, can further be used in regenerative medicine, seizing the paracrine effect of iPSCs while avoiding the possible risk of tumorigenesis associated with iPSC-based therapy [160]. The paracrine role of iPSCs in salivary gland regeneration has been proven upon coculturing embryonic submandibular gland cells and mouse iPSCs [124]. Besides, human iPSC CM promoted the osteogenic differentiation of human MSCs [122]. Usage of iPSC-derived secretome for tissue regeneration merits further research such as determining the active genes and growth factors expressed in CM from iPSCs.

Defining the optimum and the most accessible cell source to attain iPSCs should be investigated in the future to maximize their differentiation potential as well as their generation efficacy. iPSCs proved to retain their epigenetic memory, which may affect their subsequent differentiation [155, 156]. For example, iPSCs derived from gingival fibroblasts showed a higher expression of periodontal cell markers in vitro [125]. This could be beneficial in using particular cell sources for specific tissue regeneration, but it hinders the wide range of cells that could be derived from iPSCs. Despite that gingival fibroblasts and urine cells could be considered an easy source for attaining iPSCs, the generation efficiency of iPSCs using fibroblasts is extremely low [151].

A better control of the differentiation potential of iPSCs could be achieved by defining the suspension time of EB, since iPSCs-MSCs obtained at early EB suspension time possessed a more stem cell phenotype while those cells obtained later acquired a more differentiated phenotype [68], and by controlling and optimizing the reprogramming method where the highest cell density was attained in Sendai-iPSCs, while retro-iPSCs showed poor osteogenic differentiation [86].

Finally, next-generation sequencing could be alternatively used to assess the pluripotency potential, following iPSC generation instead of the complicated current techniques including teratoma formation and in vitro embryoid body (EB) generation [32].

\section{Conclusion}

iPSCs represent an autologous cell source, derived from the patient's own tissue, with no risk of immune reaction [161163]. They have higher proliferative rates than adult stem cells and can be acquired via noninvasive methods [161], all properties that are highly desirable in regenerative medicine. Despite challenges associated with iPSCs' clinical use, their potential impact on medical applications still warrants further research. Carrying the application of iPSCs for tissue regeneration into humans entails strict abiding to the conduct of good manufacturing practice (GMP), as well as properly selecting cell source, culturing media, and vectors for gene transduction and excluding any xenogeneic-derived products from iPSC generation protocols. Recently, iPSCs have been successfully generated using a protocol compliant with GMP from hematopoietic stem cells from peripheral blood [164]. Furthermore, iPSCs were successfully maintained undifferentiated in xenogeneic-free culture medium 
and were subsequently differentiated into MSCs and osteogenic cells. Positive results were also attained following implantation in rats' calvarial defects [165] which paves the way for carrying iPSCs into clinical trials. Initial reports documented that the risk of teratoma formation associated with iPSC transplantation could be inhibited by pretreatment with resveratrol [112] or by irradiation of 2 Gray (Gy) prior to transplantation [43]. Finally, iPSCs' extracellular vesicles and secretomes, containing protein, mRNA, and miRNA, can alternatively be used, exploiting the paracrine effect of iPSCs while avoiding the risk of tumorigenesis associated with iPSC-based therapy [160].

It can thus be concluded that even though iPSCs hold a tremendously unexplored potential in the field of regenerative medicine, bone and dental tissue engineering, therapeutic application in bone disorders, gene therapy, and personalized medicine, a number of obstacles must be alleviated to attain their clinical applications. iPSCs still warrant further research focusing on achieving a safe, efficient reprogramming and attaining significant expansion while evading postimplantation tumor risks. Unleashing the full capabilities of iPSCs holds a promise of offering remedies to several genetic disorders in addition to their potential application in bone and dental tissue regeneration.

\section{Abbreviations}

ALP:

ASC-iPSCs:

AT-MSCs:

bFGF:

BM-MSCs:

BMP:

BMSC:

CA:

CCD:

CCHS:

CM:

CMC:

Col2.3GFP:

CPC:

DFs:

DPI-VTK:

EBs:

EF:

ELF-EMF:

EMD:

ES:

G/C/GP:

GDF-5:

$\mathrm{GO}$ :

HA/Col/CTS:

HA/TCP:

$\mathrm{HA}$ :

HCG:
Alkaline phosphatase

Adipose-derived induced pluripotent

stem cells

Human adipose tissue

Basic fibroblast growth factor

Bone marrow mesenchymal stem cells

Bone morphogenetic protein

Bone marrow stromal cells

Cytomegalovirus enhancer/b-actin

Collagen/chitosan/hydroxyapatite

scaffolds

Conditioned media

Carboxymethyl chitosan

$2.3 \mathrm{~kb}$ type I collagen promoter-driven green fluorescent protein

Calcium phosphate cement

Dermal fibroblasts

Dpiyalswsgma-Vtkhlnqisqsy

Embryoid bodies

Embryonic fibroblasts

Extremely low-frequency electromagnetic field

Enamel matrix derivatives

Embryonic stem

Chitosan/gelatin/glycerol phosphate

Growth/differentiation factor-5

Graphene oxide

Hydroxyapatite/collagen/chitosan

Hydroxyapatite/tricalcium phosphate

Hydroxyapatite

Nanohydroxyapatite/chitosan/gelatin
Cleidocranial dysostosis
HEK: Human embryonic kidney

hiPSCs: Human induced pluripotent stem cells

HUVECs: Human umbilical vein endothelial cells

iPSCs: Induced pluripotent stem cells

iPS-NC-PDL cells: iPSCs induced into neural crest- (NC-) like cells

iPS-NC cells: $\quad$ p75 neurotrophic receptor-positive cells were cultured on extracellular matrix (ECM) produced by human PDL

MSCs: Mesenchymal stem cells

NCLCs: Neural crest-like cells

nHA/CG scaffolds: Nanohydroxyapatite/chitosan/gelatin scaffolds

nHA: Nanohydroxyapatite

OM: $\quad$ Osteogenic media

PCL: $\quad$ Polycaprolactone

PCL-PLLA: Polycaprolactone-poly-L-lactic acid

PCL-PVDF: $\quad$ Polycaprolactone-polyvinylidene fluoride

pDA: $\quad$ Polydopamine

PBMC: $\quad$ Peripheral blood mononuclear cell

PDL: $\quad$ Periodontal ligaments

PEG: $\quad$ Polyethylene glycol

PES: $\quad$ Polyethersulfone

PHT: $\quad$ Polymer hyaluronan and ceramic tricalcium phosphate ceramic particles

PLA: $\quad$ Poly-L-lactic acid

PLCL: Poly (L-lactic acid-co- $\varepsilon$-caprolactone)

PLGA/PLLA: Poly lactic-co-glycolic acid/poly L-lactic acid

Poly-P: Polyphosphate

PVDF: $\quad$ Polyvinylidene fluoride

PVDF/Col/PRP: Polyvinylidene fluoride/collagen/platelet-rich plasma

RGD: Arg-Gly-Asp

RUNX2: $\quad$ Runt-related transcription factor 2

SHED: Human exfoliated deciduous teeth

TGF- $\beta$ : Transforming growth factor-beta

TNAP: Tissue-nonspecific alkaline phosphatase

TSG-6: $\quad$ Tumor necrosis factor alpha-stimulated gene-6.

\section{Conflicts of Interest}

The authors declare that they have no conflicts of interest.

\section{References}

[1] M. J. Evans and M. H. Kaufman, "Establishment in culture of pluripotential cells from mouse embryos," Nature, vol. 292, no. 5819 , pp. $154-156,1981$.

[2] L. Gepstein, "Derivation and potential applications of human embryonic stem cells," Circulation Research, vol. 91, no. 10, pp. 866-876, 2002.

[3] G. R. Martin, "Isolation of a pluripotent cell line from early mouse embryos cultured in medium conditioned by teratocarcinoma stem cells," Proceedings of the National Academy of Sciences of the United States of America, vol. 78, no. 12, pp. 7634-7638, 1981. 
[4] J. A. Thomson, J. Itskovitz-Eldor, S. S. Shapiro et al., "Embryonic stem cell lines derived from human blastocysts," Science, vol. 282, no. 5391, pp. 1145-1147, 1998.

[5] Y.-C. Chen, K.-L. Tsai, C.-W. Hung et al., "Induced pluripotent stem cells and regenerative medicine," Journal of Clinical Gerontology and Geriatrics, vol. 2, no. 1, article S2210833510000389, pp. 1-6, 2011.

[6] K. Takahashi and S. Yamanaka, "Induction of pluripotent stem cells from mouse embryonic and adult fibroblast cultures by defined factors," Cell, vol. 126, no. 4, pp. 663-676, 2006.

[7] E. E. Morales and R. A. Wingert, "Renal stem cell reprogramming: prospects in regenerative medicine," World Journal of Stem Cells, vol. 6, no. 4, pp. 458-466, 2014.

[8] S. Zhu, W. Li, H. Zhou et al., "Reprogramming of human primary somatic cells by OCT4 and chemical compounds," Cell Stem Cell, vol. 7, no. 6, pp. 651-655, 2010.

[9] K. Takahashi, K. Tanabe, M. Ohnuki et al., "Induction of pluripotent stem cells from adult human fibroblasts by defined factors," Cell, vol. 131, no. 5, pp. 861-872, 2007.

[10] B. Ebrahimi, "Reprogramming of adult stem/progenitor cells into iPSCs without reprogramming factors," Journal of Medical Hypotheses and Ideas, vol. 9, no. 2, pp. 99-103, 2015.

[11] E. Kiskinis and K. Eggan, "Progress toward the clinical application of patient-specific pluripotent stem cells," The Journal of Clinical Investigation, vol. 120, no. 1, pp. 5159, 2010.

[12] S. Raab, M. Klingenstein, S. Liebau, and L. Linta, "A comparative view on human somatic cell sources for iPSC generation," Stem Cells International, vol. 2014, Article ID 768391, 12 pages, 2014.

[13] N. Wada, B. Wang, N. H. Lin, A. L. Laslett, S. Gronthos, and P. M. Bartold, "Induced pluripotent stem cell lines derived from human gingival fibroblasts and periodontal ligament fibroblasts," Journal of Periodontal Research, vol. 46, no. 4, pp. 438-447, 2011.

[14] H. Egusa, K. Okita, H. Kayashima et al., "Gingival fibroblasts as a promising source of induced pluripotent stem cells," PLoS One, vol. 5, no. 9, article e12743, 2010.

[15] Y. Nomura, M. Ishikawa, Y. Yashiro et al., "Human periodontal ligament fibroblasts are the optimal cell source for induced pluripotent stem cells," Histochemistry and Cell Biology, vol. 137, no. 6, pp. 719-732, 2012.

[16] K. Otsu, M. Kumakami-Sakano, N. Fujiwara et al., "Stem cell sources for tooth regeneration: current status and future prospects," Frontiers in Physiology, vol. 5, p. 36, 2014.

[17] N. Tamaoki, K. Takahashi, T. Tanaka et al., "Dental pulp cells for induced pluripotent stem cell banking," Journal of Dental Research, vol. 89, no. 8, pp. 773-778, 2010.

[18] X. Yan, H. Qin, C. Qu, R. S. Tuan, S. Shi, and G. T. J. Huang, "iPS cells reprogrammed from human mesenchymal-like stem/progenitor cells of dental tissue origin," Stem Cells and Development, vol. 19, no. 4, pp. 469-480, 2010.

[19] P. C. B. Beltrão-Braga, G. C. Pignatari, P. C. Maiorka et al., "Feeder-free derivation of induced pluripotent stem cells from human immature dental pulp stem cells," Cell Transplantation, vol. 20, no. 11-12, pp. 1707-1719, 2011.

[20] K. Okita, M. Nakagawa, H. Hyenjong, T. Ichisaka, and S. Yamanaka, "Generation of mouse induced pluripotent stem cells without viral vectors," Science, vol. 322, no. 5903, pp. 949-953, 2008.
[21] W. Deng, "Induced pluripotent stem cells: paths to new medicines. A catalyst for disease modelling, drug discovery and regenerative therapy," EMBO Reports, vol. 11, no. 3, pp. 161-165, 2010.

[22] S. P. Medvedev, A. I. Shevchenko, and S. M. Zakian, "Induced pluripotent stem cells: problems and advantages when applying them in regenerative medicine," Acta Naturae, vol. 2, no. 2, pp. 18-27, 2010.

[23] S. Romanazzo, S. Nemec, and I. Roohani, "iPSC bioprinting: where are we at?," Materials, vol. 12, no. 15, article 2453, 2019.

[24] K. Tashiro, M. Inamura, K. Kawabata et al., "Efficient adipocyte and osteoblast differentiation from mouse induced pluripotent stem cells by adenoviral transduction," Stem Cells, vol. 27, no. 8, pp. 1802-1811, 2009.

[25] D. Kim, C.-H. Kim, J.-I. Moon et al., "Generation of human induced pluripotent stem cells by direct delivery of reprogramming proteins," Cell Stem Cell, vol. 4, no. 6, pp. 472476, 2009.

[26] L. Warren, P. D. Manos, T. Ahfeldt et al., "Highly efficient reprogramming to pluripotency and directed differentiation of human cells with synthetic modified mRNA," Cell Stem Cell, vol. 7, no. 5, pp. 618-630, 2010.

[27] F. Anokye-Danso, C. M. Trivedi, D. Juhr et al., "Highly efficient miRNA-mediated reprogramming of mouse and human somatic cells to pluripotency," Cell Stem Cell, vol. 8, no. 4, pp. 376-388, 2011.

[28] N. Miyoshi, H. Ishii, H. Nagano et al., "Reprogramming of mouse and human cells to pluripotency using mature microRNAs," Cell Stem Cell, vol. 8, no. 6, pp. 633-638, 2011.

[29] B. Saha, M. P. Borgohain, C. Dey, and R. P. Thummer, "iPS cell generation: current and future challenges," Annals of Stem Cell Research \& Therapy, vol. 1, no. 2, p. 1007, 2018.

[30] N. Malik and M. S. Rao, "A review of the methods for human iPSC derivation," Methods in Molecular Biology, vol. 997, pp. 23-33, 2013.

[31] P. Czerwinska, S. Mazurek, I. Kolodziejczak, and M. Wiznerowicz, "Gene delivery methods and genome editing of human pluripotent stem cells," Reports of Practical Oncology and Radiotherapy, vol. 24, no. 2, pp. 180-187, 2019.

[32] R. Jaenisch and R. Young, "Stem cells, the molecular circuitry of pluripotency and nuclear reprogramming," Cell, vol. 132, no. 4, pp. 567-582, 2008.

[33] S. D. Sheridan, V. Surampudi, and R. R. Rao, "Analysis of embryoid bodies derived from human induced pluripotent stem cells as a means to assess pluripotency," Stem Cells International, vol. 2012, Article ID 738910, 9 pages, 2012.

[34] H. Kurosawa, "Methods for inducing embryoid body formation: in vitro differentiation system of embryonic stem cells," Journal of Bioscience and Bioengineering, vol. 103, no. 5, pp. 389-398, 2007.

[35] G. Pettinato, X. Wen, and N. Zhang, "Formation of welldefined embryoid bodies from dissociated human induced pluripotent stem cells using microfabricated cell-repellent microwell arrays," Scientific Reports, vol. 4, no. 1, article $7402,2014$.

[36] J. Itskovitz-Eldor, M. Schuldiner, D. Karsenti et al., "Differentiation of human embryonic stem cells into embryoid bodies 
comprising the three embryonic germ layers," Molecular Medicine, vol. 6, no. 2, pp. 88-95, 2000.

[37] A. J. Joannides, C. Fiore-Hériché, A. A. Battersby et al., "A scaleable and defined system for generating neural stem cells from human embryonic stem cells," Stem Cells, vol. 25, no. 3, pp. 731-737, 2007.

[38] J. C. Mohr, J. Zhang, S. M. Azarin et al., "The microwell control of embryoid body size in order to regulate cardiac differentiation of human embryonic stem cells," Biomaterials, vol. 31, no. 7, pp. 1885-1893, 2010.

[39] B. Valamehr, S. J. Jonas, J. Polleux et al., "Hydrophobic surfaces for enhanced differentiation of embryonic stem cellderived embryoid bodies," Proceedings of the National Academy of Sciences of the United States of America, vol. 105, no. 38, pp. 14459-14464, 2008.

[40] A. Sakkas, F. Wilde, M. Heufelder, K. Winter, and A. Schramm, "Autogenous bone grafts in oral implantology—is it still a "gold standard"? A consecutive review of 279 patients with 456 clinical procedures," International Journal of Implant Dentistry, vol. 3, no. 1, p. 23, 2017.

[41] Z. Sheikh, N. Hamdan, Y. Ikeda, M. Grynpas, B. Ganss, and M. Glogauer, "Natural graft tissues and synthetic biomaterials for periodontal and alveolar bone reconstructive applications: a review," Biomaterials Research, vol. 21, no. 1, p. 9, 2017.

[42] D. Rana, S. Kumar, T. J. Webster, and M. Ramalingam, "Impact of induced pluripotent stem cells in bone repair and regeneration," Current Osteoporosis Reports, vol. 17, no. 4, pp. 226-234, 2019.

[43] T. Hayashi, H. Misawa, H. Nakahara et al., "Transplantation of osteogenically differentiated mouse iPS cells for bone repair," Cell Transplantation, vol. 21, no. 2-3, pp. 591-600, 2012.

[44] S. T. Kumaran, K. V. Arun, S. Sudarsan, A. Talwar, and N. Srinivasan, "Osteoblast response to commercially available demineralized bone matrices-an in-vitro study," Indian Journal of Dental Research, vol. 21, no. 1, pp. 3-9, 2010.

[45] N. Quarto, S. Li, A. Renda, and M. T. Longaker, "Exogenous activation of BMP-2 signaling overcomes TGF $\beta$-Mediated inhibition of osteogenesis in Marfan embryonic stem cells and Marfan patient-specific induced pluripotent stem cells," Stem Cells, vol. 30, no. 12, pp. 2709-2719, 2012.

[46] N. I. zur Nieden, G. Kempka, and H. J. Ahr, "In vitro differentiation of embryonic stem cells into mineralized osteoblasts," Differentiation, vol. 71, no. 1, pp. 18-27, 2003.

[47] M. F. Abazari, F. Soleimanifar, S. E. Enderami et al., "Incorporated-bFGF polycaprolactone/polyvinylidene fluoride nanocomposite scaffold promotes human induced pluripotent stem cells osteogenic differentiation," Journal of Cellular Biochemistry, vol. 120, no. 10, pp. 16750-16759, 2019.

[48] A. Ardeshirylajimi, S. Hosseinkhani, K. Parivar, P. Yaghmaie, and M. Soleimani, "Nanofiber-based polyethersulfone scaffold and efficient differentiation of human induced pluripotent stem cells into osteoblastic lineage," Molecular Biology Reports, vol. 40, no. 7, pp. 4287-4294, 2013.

[49] J. Ji, X. Tong, X. Huang, J. Zhang, H. Qin, and Q. Hu, "Patient-derived human induced pluripotent stem cells from gingival fibroblasts composited with defined nanohydroxyapatite/chitosan/gelatin porous scaffolds as potential bone graft substitutes," Stem Cells Translational Medicine, vol. 5, no. 1, pp. 95-105, 2016.
[50] A. Tahmasebi, S. E. Enderami, E. Saburi et al., "Micro-RNAincorporated electrospun nanofibers improve osteogenic differentiation of human-induced pluripotent stem cells," Journal of Biomedical Materials Research Part A, vol. 108, no. 2, pp. 377-386, 2019.

[51] M.-S. Ma, V. Kannan, A. E. de Vries et al., "Characterization and comparison of osteoblasts derived from mouse embryonic stem cells and induced pluripotent stem cells," Journal of Bone and Mineral Metabolism, vol. 35, no. 1, pp. 21-30, 2017.

[52] A. Saito, A. Ooki, T. Nakamura et al., "Targeted reversion of induced pluripotent stem cells from patients with human cleidocranial dysplasia improves bone regeneration in a rat calvarial bone defect model," Stem Cell Research \& Therapy, vol. 9, no. 1, p. 12, 2018.

[53] A. Ardeshirylajimi, P. Dinarvand, E. Seyedjafari, L. Langroudi, F. Jamshidi Adegani, and M. Soleimani, "Enhanced reconstruction of rat calvarial defects achieved by plasma-treated electrospun scaffolds and induced pluripotent stem cells," Cell and Tissue Research, vol. 354, no. 3, pp. 849-860, 2013.

[54] H. Zhu, T. Kimura, S. Swami, and J. Y. Wu, "Pluripotent stem cells as a source of osteoblasts for bone tissue regeneration," Biomaterials, vol. 196, pp. 31-45, 2019.

[55] R. Xu, Z. Zhang, M. S. Toftdal et al., "Synchronous delivery of hydroxyapatite and connective tissue growth factor derived osteoinductive peptide enhanced osteogenesis," Journal of Controlled Release, vol. 301, pp. 129-139, 2019.

[56] K. Hayashi, H. Ochiai-Shino, T. Shiga et al., "Transplantation of human-induced pluripotent stem cells carried by selfassembling peptide nanofiber hydrogel improves bone regeneration in rat calvarial bone defects," BDJ Open, vol. 2, no. 1, article 15007, 2016.

[57] J.-Y. Ko, S. Park, and G.-I. Im, “Osteogenesis from human induced pluripotent stem cells: an in vitro and in vivo comparison with mesenchymal stem cells," Stem Cells and Development, vol. 23, no. 15, pp. 1788-1797, 2014.

[58] T.-J. Lee, J. Jang, S. Kang et al., "Mesenchymal stem cellconditioned medium enhances osteogenic and chondrogenic differentiation of human embryonic stem cells and human induced pluripotent stem cells by mesodermal lineage induction," Tissue Engineering Part A, vol. 20, no. 7-8, pp. 13061313, 2014.

[59] S. Zhong, X. He, Y. Li, and X. Lou, "Conditioned medium enhances osteogenic differentiation of induced pluripotent stem cell-derived mesenchymal stem cells," Tissue Engineering and Regenerative Medicine, vol. 16, no. 2, pp. 141-150, 2019.

[60] F. Li, S. Bronson, and C. Niyibizi, "Derivation of murine induced pluripotent stem cells (iPS) and assessment of their differentiation toward osteogenic lineage," Journal of Cellular Biochemistry, vol. 109, no. 4, pp. 643-652, 2010.

[61] F. Li and C. Niyibizi, "Cells derived from murine induced pluripotent stem cells (iPSC) by treatment with members of TGF-beta family give rise to osteoblasts differentiation and form bone in vivo," BMC Cell Biology, vol. 13, no. 1, p. 35, 2012.

[62] P. Wang, T. Ma, D. Guo et al., "Metformin induces osteoblastic differentiation of human induced pluripotent stem cellderived mesenchymal stem cells," Journal of Tissue Engineering and Regenerative Medicine, vol. 12, no. 2, pp. 437-446, 2018. 
[63] W. Chen, X. Liu, Q. Chen et al., "Angiogenic and osteogenic regeneration in rats via calcium phosphate scaffold and endothelial cell co-culture with human bone marrow mesenchymal stem cells (MSCs), human umbilical cord MSCs, human induced pluripotent stem cell-derived MSCs and human embry," Journal of Tissue Engineering and Regenerative Medicine, vol. 12, no. 1, pp. 191-203, 2018.

[64] X. Liu, W. Chen, C. Zhang et al., "Co-seeding human endothelial cells with human-induced pluripotent stem cellderived mesenchymal stem cells on calcium phosphate scaffold enhances osteogenesis and vascularization in rats," Tissue Engineering Part A, vol. 23, no. 11-12, pp. 546-555, 2017.

[65] C. Zhang, K. Hu, X. Liu et al., "Novel hiPSC-based tri-culture for pre-vascularization of calcium phosphate scaffold to enhance bone and vessel formation," Materials Science \& Engineering C, Materials for Biological Applications, vol. 79, pp. 296-304, 2017.

[66] J. Liu, W. Chen, Z. Zhao, and H. H. K. Xu, "Reprogramming of mesenchymal stem cells derived from iPSCs seeded on biofunctionalized calcium phosphate scaffold for bone engineering," Biomaterials, vol. 34, no. 32, pp. 7862-7872, 2013.

[67] J. Liu, W. Chen, Z. Zhao, and H. H. K. Xu, "Effect of NELL1 gene overexpression in iPSC-MSCs seeded on calcium phosphate cement," Acta Biomaterialia, vol. 10, no. 12, pp. 5128$5138,2014$.

[68] D. Sheyn, S. Ben-David, G. Shapiro et al., "Human induced pluripotent stem cells differentiate into functional mesenchymal stem cells and repair bone defects," Stem Cells Translational Medicine, vol. 5, no. 11, pp. 1447-1460, 2016.

[69] P. Wang, Y. Song, M. D. Weir et al., "A self-setting iPSMSCalginate-calcium phosphate paste for bone tissue engineering," Dental Materials, vol. 32, no. 2, pp. 252-263, 2016.

[70] Q. Wu, B. Yang, C. Cao, K. Hu, P. Wang, and Y. Man, “Therapeutic antibody directed osteogenic differentiation of induced pluripotent stem cell derived MSCs," Acta Biomaterialia, vol. 74, pp. 222-235, 2018.

[71] H. Ramaraju and D. H. Kohn, "Cell and material-specific phage display peptides increase iPS-MSC mediated bone and vasculature formation in vivo," Advanced Healthcare Materials, vol. 8, no. 9, article e1801356, 2019.

[72] W. TheinHan, J. Liu, M. Tang, W. Chen, L. Cheng, and H. H. $\mathrm{K}$. Xu, "Induced pluripotent stem cell-derived mesenchymal stem cell seeding on biofunctionalized calcium phosphate cements," Bone Research, vol. 1, no. 4, pp. 371-384, 2013.

[73] P. Wang, X. Liu, L. Zhao et al., "Bone tissue engineering via human induced pluripotent, umbilical cord and bone marrow mesenchymal stem cells in rat cranium," Acta Biomaterialia, vol. 18, pp. 236-248, 2015.

[74] J. Xie, C. Peng, Q. Zhao et al., "Osteogenic differentiation and bone regeneration of iPSC-MSCs supported by a biomimetic nanofibrous scaffold," Acta Biomaterialia, vol. 29, pp. 365379, 2016.

[75] A. Al-Wahabi, T. Ser-Od, K. Inoue, K. Nakajima, K. Matsuzaka, and T. Inoue, "Topography enhances Runx 2 expression in outgrowing cells from iPS cell-derived embryoid bodies," Journal of Biomedical Materials Research Part B: Applied Biomaterials, vol. 107, no. 7, pp. 2288-2296, 2019.

[76] M. Sladkova, M. Palmer, C. Öhman et al., "Fabrication of macroporous cement scaffolds using PEG particles: in vitro evaluation with induced pluripotent stem cell-derived mesenchymal progenitors," Materials Science \& Engineering C,
Materials for Biological Applications, vol. 69, pp. 640-652, 2016.

[77] M. Tang, W. Chen, J. Liu, M. D. Weir, L. Cheng, and H. H. K. $\mathrm{Xu}$, "Human induced pluripotent stem cell-derived mesenchymal stem cell seeding on calcium phosphate scaffold for bone regeneration," Tissue Engineering Part A, vol. 20, no. 7-8, pp. 1295-1305, 2014.

[78] L. Wang, P. Wang, M. D. Weir, M. A. Reynolds, L. Zhao, and H. H. K. Xu, "Hydrogel fibers encapsulating human stem cells in an injectable calcium phosphate scaffold for bone tissue engineering," Biomedical Materials, vol. 11, no. 6, article 65008, 2016.

[79] L. Wang, C. Zhang, C. Li et al., "Injectable calcium phosphate with hydrogel fibers encapsulating induced pluripotent, dental pulp and bone marrow stem cells for bone repair," Materials Science \& Engineering C, Materials for Biological Applications, vol. 69, pp. 1125-1136, 2016.

[80] O. H. Jeon, L. M. Panicker, Q. Lu, J. J. Chae, R. A. Feldman, and J. H. Elisseeff, "Human iPSC-derived osteoblasts and osteoclasts together promote bone regeneration in 3D biomaterials," Scientific Reports, vol. 6, no. 1, article 26761, 2016.

[81] G. Bilousova, D. H. Jun, K. B. King et al., "Osteoblasts derived from induced pluripotent stem cells form calcified structures in scaffolds both in vitro and in vivo," Stem Cells, vol. 29, no. 2, pp. 206-216, 2011.

[82] A. L. Firth and J. X. J. Yuan, ““"Ether-à-go-go” proliferation of iPSC-derived mesenchymal stem cells. Focus on "Regulation of cell proliferation of human induced pluripotent stem cellderived mesenchymal stem cells via ether-à-go-go 1 (hEAG1) potassium channel"," American Journal of Physiology-Cell Physiology, vol. 303, no. 2, pp. C113-C114, 2012.

[83] R. M. Guzzo, J. Gibson, R. H. Xu, F. Y. Lee, and H. Drissi, "Efficient differentiation of human iPSC-derived mesenchymal stem cells to chondroprogenitor cells," Journal of Cellular Biochemistry, vol. 114, no. 2, pp. 480-490, 2013.

[84] Q. Lian, Y. Zhang, J. Zhang et al., "Functional mesenchymal stem cells derived from human induced pluripotent stem cells attenuate limb ischemia in mice," Circulation, vol. 121, no. 9, pp. 1113-1123, 2010.

[85] J. Zhang, J. Guan, X. Qi et al., "Dimethyloxaloylglycine promotes the angiogenic activity of mesenchymal stem cells derived from iPSCs via activation of the PI3K/Akt pathway for bone regeneration," International Journal of Biological Sciences, vol. 12, no. 6, pp. 639-652, 2016.

[86] G. M. de Peppo, I. Marcos-Campos, D. J. Kahler et al., "Engineering bone tissue substitutes from human induced pluripotent stem cells," Proceedings of the National Academy of Sciences of the United States of America, vol. 110, no. 21, pp. 8680-8685, 2013.

[87] S. G. Hong, T. Winkler, C. Wu et al., "Path to the clinic: assessment of iPSC-based cell therapies in vivo in a nonhuman primate model," Cell Reports, vol. 7, no. 4, pp. 12981309, 2014.

[88] K. Hynes, D. Menicanin, K. Mrozik, S. Gronthos, and P. M. Bartold, "Generation of functional mesenchymal stem cells from different induced pluripotent stem cell lines," Stem Cells and Development, vol. 23, no. 10, pp. 1084-1096, 2014.

[89] R. Kang, Y. Luo, L. Zou et al., "Osteogenesis of human induced pluripotent stem cells derived mesenchymal stem cells on hydroxyapatite contained nanofibers," RSC Advances, vol. 4, no. 11, pp. 5734-5739, 2014. 
[90] L. Zou, Y. Luo, M. Chen et al., "A simple method for deriving functional MSCs and applied for osteogenesis in 3D scaffolds," Scientific Reports, vol. 3, no. 1, article 2243, 2013.

[91] A. Nasu, M. Ikeya, T. Yamamoto et al., "Genetically matched human iPS cells reveal that propensity for cartilage and bone differentiation differs with clones, not cell type of origin," PLoS One, vol. 8, no. 1, article e53771, 2013.

[92] R. Chijimatsu, M. Ikeya, Y. Yasui et al., "Characterization of mesenchymal stem cell-like cells derived from human iPSCs via neural crest development and their application for osteochondral repair," Stem Cells International, vol. 2017, Article ID 1960965, 18 pages, 2017.

[93] Y. Dogaki, S. Y. Lee, T. Niikura et al., "Efficient derivation of osteoprogenitor cells from induced pluripotent stem cells for bone regeneration," International Orthopaedics, vol. 38, no. 9, pp. 1779-1785, 2014.

[94] F. A. A. Ishiy, R. D. Fanganiello, K. Griesi-Oliveira et al., "Improvement of in vitro osteogenic potential through differentiation of induced pluripotent stem cells from human exfoliated dental tissue towards mesenchymal-like stem cells," Stem Cells International, vol. 2015, Article ID 249098, 9 pages, 2015.

[95] R. Kang, Y. Zhou, S. Tan et al., "Mesenchymal stem cells derived from human induced pluripotent stem cells retain adequate osteogenicity and chondrogenicity but less adipogenicity," Stem Cell Research and Therapy, vol. 6, no. 1, 2015.

[96] S. I. Lepage, K. Nagy, H. K. Sung, R. A. Kandel, A. Nagy, and T. G. Koch, "Generation, characterization, and multilineage potency of mesenchymal-like progenitors derived from equine induced pluripotent stem cells," Stem Cells and Development, vol. 25, no. 1, pp. 80-89, 2016.

[97] A. Ardeshirylajimi, M. Soleimani, S. Hosseinkhani, K. Parivar, and P. Yaghmaei, "A comparative study of osteogenic differentiation human induced pluripotent stem cells and adipose tissue derived mesenchymal stem cells," Cell Journal, vol. 16, no. 3, pp. 235-244, 2014.

[98] S.-H. Mao, C.-H. C.-T. Chen, and C.-H. C.-T. Chen, “Osteogenic potential of induced pluripotent stem cells from human adipose-derived stem cells," Stem Cell Research \& Therapy, vol. 10, no. 1, p. 303, 2019.

[99] M. D. Phillips, S. A. Kuznetsov, N. Cherman et al., "Directed differentiation of human induced pluripotent stem cells toward bone and cartilage: in vitro versus in vivo assays," Stem Cells Translational Medicine, vol. 3, no. 7, pp. 867878, 2014.

[100] B. Levi, J. S. Hyun, D. T. Montoro et al., "In vivo directed differentiation of pluripotent stem cells for skeletal regeneration," Proceedings of the National Academy of Sciences of the United States of America, vol. 109, no. 50, pp. 20379-20384, 2012.

[101] Y. Deng, Y. Yang, and S. Wei, "Peptide-decorated nanofibrous niche augments in vitro directed osteogenic conversion of human pluripotent stem cells," Biomacromolecules, vol. 18, no. 2, pp. 587-598, 2017.

[102] H. Kang, Y.-R. V. Shih, M. Nakasaki, H. Kabra, and S. Varghese, "Small molecule-driven direct conversion of human pluripotent stem cells into functional osteoblasts," Science Advances, vol. 2, no. 8, article e1600691, 2016.

[103] S. Kawai, H. Yoshitomi, J. Sunaga et al., "In vitro bone-like nodules generated from patient-derived iPSCs recapitulate pathological bone phenotypes," Nature Biomedical Engineering, vol. 3, no. 7, pp. 558-570, 2019.
[104] J.-H. Ye, Y.-J. Xu, J. Gao et al., “Critical-size calvarial bone defects healing in a mouse model with silk scaffolds and SATB2-modified iPSCs," Biomaterials, vol. 32, no. 22, pp. 5065-5076, 2011.

[105] M. Wang, Y. Deng, P. Zhou et al., "In vitro culture and directed osteogenic differentiation of human pluripotent stem cells on peptides-decorated two-dimensional microenvironment," ACS Applied Materials \& Interfaces, vol. 7, no. 8, pp. 4560-4572, 2015.

[106] G.-Z. Jin, T.-H. Kim, J.-H. Kim et al., "Bone tissue engineering of induced pluripotent stem cells cultured with macrochanneled polymer scaffold," Journal of Biomedical Materials Research Part A, vol. 101, no. 5, pp. 1283-1291, 2013.

[107] H. Kang, Y.-R. V. Shih, Y. Hwang et al., "Mineralized gelatin methacrylate-based matrices induce osteogenic differentiation of human induced pluripotent stem cells," Acta Biomaterialia, vol. 10, no. 12, pp. 4961-4970, 2014.

[108] M. Sladkova, J. Cheng, M. Palmer et al., "Comparison of decellularized cow and human bone for engineering bone grafts with human induced pluripotent stem cells," Tissue Engineering Part A, vol. 25, no. 3-4, pp. 288-301, 2019.

[109] H. Ochiai-Shino, H. Kato, T. Sawada et al., "A novel strategy for enrichment and isolation of osteoprogenitor cells from induced pluripotent stem cells based on surface marker combination.," PLoS One, vol. 9, no. 6, article e99534, 2014.

[110] A. Ardeshirylajimi and M. Soleimani, "Enhanced growth and osteogenic differentiation of induced pluripotent stem cells by extremely low-frequency electromagnetic field," Cellular and Molecular Biology, vol. 61, no. 1, pp. 36-41, 2015.

[111] S. Das and D. K. Das, "Anti-inflammatory responses of resveratrol," Inflammation \& Allergy Drug Targets, vol. 6, no. 3, pp. 168-173, 2007.

[112] C.-L. Kao, L.-K. Tai, S.-H. Chiou et al., "Resveratrol promotes osteogenic differentiation and protects against dexamethasone damage in murine induced pluripotent stem cells," Stem Cells and Development, vol. 19, no. 2, pp. 247-258, 2010.

[113] J. Park, J. Ries, K. Gelse et al., "Bone regeneration in critical size defects by cell-mediated BMP-2 gene transfer: a comparison of adenoviral vectors and liposomes," Gene Therapy, vol. 10, no. 13, pp. 1089-1098, 2003.

[114] K. Kanke, H. Masaki, T. Saito et al., "Stepwise differentiation of pluripotent stem cells into osteoblasts using four small molecules under serum-free and feeder-free conditions," Stem Cell Reports, vol. 2, no. 6, pp. 751-760, 2014.

[115] Y. Wu, H. Sun, F. Song, C. Huang, and J. Wang, "Deletion of Alox 5 gene decreases osteogenic differentiation but increases adipogenic differentiation of mouse induced pluripotent stem cells," Cell and Tissue Research, vol. 358, no. 1, pp. 135-147, 2014.

[116] A. Mirzaei, A. S. Moghadam, M. F. Abazari et al., "Comparison of osteogenic differentiation potential of induced pluripotent stem cells on $2 \mathrm{D}$ and $3 \mathrm{D}$ polyvinylidene fluoride scaffolds," Journal of Cellular Physiology, vol. 234, no. 10, pp. 17854-17862, 2019.

[117] F. S. Hosseini, F. Soleimanifar, A. Aidun et al., "Poly (3hydroxybutyrate-co-3-hydroxyvalerate) improved osteogenic differentiation of the human induced pluripotent stem cells while considered as an artificial extracellular matrix," Journal of Cellular Physiology, vol. 234, no. 7, pp. 1153711544, 2019. 
[118] F. S. Hosseini, F. Soleimanifar, A. Khojasteh, and A. Ardeshirylajimi, "Promoting osteogenic differentiation of human-induced pluripotent stem cells by releasing $\mathrm{Wnt} / \beta$ catenin signaling activator from the nanofibers," Journal of Cellular Biochemistry, vol. 120, no. 4, pp. 6339-6346, 2019.

[119] E. Saburi, M. Islami, S. Hosseinzadeh et al., "In vitro osteogenic differentiation potential of the human induced pluripotent stem cells augments when grown on Graphene oxidemodified nanofibers," Gene, vol. 696, pp. 72-79, 2019.

[120] M. F. Abazari, F. Soleimanifar, M. Amini Faskhodi et al., "Improved osteogenic differentiation of human induced pluripotent stem cells cultured on polyvinylidene fluoride/collagen/platelet-rich plasma composite nanofibers," Journal of Cellular Physiology, vol. 235, no. 2, pp. 1155-1164, 2020.

[121] J. Ji, X. Tong, X. Huang et al., "Sphere-shaped nano-hydroxyapatite/chitosan/gelatin 3D porous scaffolds increase proliferation and osteogenic differentiation of human induced pluripotent stem cells from gingival fibroblasts," Biomedical Materials, vol. 10, no. 4, article 45005, 2015.

[122] K. Oudina, J. Paquet, A. Moya et al., "The paracrine effects of human induced pluripotent stem cells promote bone- like structures via the upregulation of BMP expression in a mouse ectopic model," Scientific Reports, vol. 8, no. 1, article 17106, 2018.

[123] Y. Alaa El-Din, D. Sabry, A. H. Abdelrahman, and S. Fathy, "Potential therapeutic effects of induced pluripotent stem cells on induced salivary gland cancer in experimental rats," Biotechnic \& Histochemistry, vol. 94, no. 2, pp. 92-99, 2019.

[124] H. Ono, A. Obana, Y. Usami et al., "Regenerating salivary glands in the microenvironment of induced pluripotent stem cells," BioMed Research International, vol. 2015, Article ID 293570, 11 pages, 2015.

[125] J. Li, X. Yin, and Q. Luan, “Comparative study of periodontal differentiation propensity of induced pluripotent stem cells from different tissue origins," Journal of Periodontology, vol. 89, no. 10, pp. 1230-1240, 2018.

[126] S. Hamano, A. Tomokiyo, D. Hasegawa et al., "Extracellular matrix from periodontal ligament cells could induce the differentiation of induced pluripotent stem cells to periodontal ligament stem cell-like cells," Stem cells and Development, vol. 27, no. 2, pp. 100-111, 2018.

[127] X. Duan, Q. Tu, J. Zhang et al., "Application of induced pluripotent stem (iPS) cells in periodontal tissue regeneration," Journal of Cellular Physiology, vol. 226, no. 1, pp. 150-157, 2011.

[128] X. Yin, Y. Li, J. Li et al., "Generation and periodontal differentiation of human gingival fibroblasts- derived integration-free induced pluripotent stem cells," Biochemical and Biophysical Research Communications, vol. 473, no. 3, pp. 726-732, 2016.

[129] X. Yin, P. Li, Y. Li, Y. Cai, J. Wen, and Q. Luan, "Growth/differentiation factor-5 promotes in vitro/vivo periodontal specific differentiation of induced pluripotent stem cell-derived mesenchymal stem cells," Experimental and Therapeutic Medicine, vol. 14, no. 5, pp. 4111-4117, 2017.

[130] K.-H. Chien, Y.-L. Chang, M.-L. Wang et al., "Promoting induced pluripotent stem cell-driven biomineralization and periodontal regeneration in rats with maxillary-molar defects using injectable BMP-6 hydrogel," Scientific Reports, vol. 8, no. 1, p. 114, 2018.

[131] J. W. Li, X. H. Yin, and Q. X. Luan, "Comparative study of proliferative and periodontal differentiation propensity of induced pluripotent stem cells at different passages," Beijing da xue xue bao Yi xue ban = Journal of Peking University Health sciences, vol. 49, no. 1, pp. 16-24, 2017.

[132] K. Hynes, D. Menicanin, J. Han et al., "Mesenchymal stem cells from iPS cells facilitate periodontal regeneration," Journal of Dental Research, vol. 92, no. 9, pp. 833-839, 2013.

[133] K. Hynes, R. Bright, V. Marino et al., "Potential of iPSCderived mesenchymal stromal cells for treating periodontal disease," Stem Cells International, vol. 2018, Article ID 2601945, 12 pages, 2018.

[134] H. Yang, R. M. Aprecio, X. Zhou et al., "Therapeutic effect of TSG-6 engineered iPSC-derived MSCs on experimental periodontitis in rats: a pilot study," PLoS One, vol. 9, no. 6, article e100285, 2014.

[135] M. Arakaki, M. Ishikawa, T. Nakamura et al., "Role of epithelial-stem cell interactions during dental cell differentiation," The Journal of Biological Chemistry, vol. 287, no. 13, pp. 10590-10601, 2012.

[136] K. Yoshida, J. Sato, R. Takai et al., "Differentiation of mouse iPS cells into ameloblast-like cells in cultures using medium conditioned by epithelial cell rests of Malassez and gelatincoated dishes," Medical Molecular Morphology, vol. 48, no. 3, pp. 138-145, 2015.

[137] A. N. Abdullah, S. Miyauchi, A. Onishi, K. Tanimoto, and K. Kato, "Differentiation of mouse-induced pluripotent stem cells into dental epithelial-like cells in the absence of added serum," In Vitro Cellular \& Developmental Biology Animal, vol. 55, no. 2, pp. 130-137, 2019.

[138] N. Ozeki, M. Mogi, R. Kawai et al., "Mouse-induced pluripotent stem cells differentiate into odontoblast-like cells with induction of altered adhesive and migratory phenotype of integrin," PLoS One, vol. 8, no. 11, article e80026, 2013.

[139] N. Ozeki, N. Hase, H. Yamaguchi et al., "Polyphosphate induces matrix metalloproteinase-3-mediated proliferation of odontoblast-like cells derived from induced pluripotent stem cells," Experimental Cell Research, vol. 333, no. 2, pp. 303-315, 2015.

[140] K. Otsu, R. Kishigami, A. Oikawa-Sasaki et al., "Differentiation of induced pluripotent stem cells into dental mesenchymal cells," Stem Cells and Development, vol. 21, no. 7, pp. 1156-1164, 2012.

[141] D. Seki, N. Takeshita, T. Oyanagi et al., "Differentiation of odontoblast-like cells from mouse induced pluripotent stem cells by Pax 9 and Bmp 4 transfection," Stem Cells Translational Medicine, vol. 4, no. 9, pp. 993-997, 2015.

[142] H. Xie, N. Dubey, W. Shim et al., "Functional odontoblasticlike cells derived from human iPSCs," Journal of Dental Research, vol. 97, no. 1, pp. 77-83, 2018.

[143] J. Cai, Y. Zhang, P. Liu et al., "Generation of tooth-like structures from integration-free human urine induced pluripotent stem cells," Cell Regeneration, vol. 2, no. 1, p. 2:6, 2013.

[144] L. Liu, Y.-F. Liu, J. Zhang, Y.-Z. Duan, and Y. Jin, “Ameloblasts serum-free conditioned medium: bone morphogenic protein 4-induced odontogenic differentiation of mouse induced pluripotent stem cells," Journal of Tissue Engineering and Regenerative Medicine, vol. 10, no. 6, pp. 466-474, 2016.

[145] Y. Wen, F. Wang, W. Zhang et al., "Application of induced pluripotent stem cells in generation of a tissue-engineered tooth-like structure," Tissue Engineering Part A, vol. 18, no. 15-16, pp. 1677-1685, 2012.

[146] Z. Liu, G. Zeng, Q. Qin, C. Sun, and L. Tan, "Smad 1/5 signal transduction regulates the ameloblastic differentiation of 
induced pluripotent stem cells," Brazilian Oral Research, vol. 34, article e006, 2020.

[147] S. Nori, Y. Okada, S. Nishimura et al., "Long-term safety issues of iPSC-based cell therapy in a spinal cord injury model: oncogenic transformation with epithelialmesenchymal transition," Stem Cell Reports, vol. 4, no. 3, pp. 360-373, 2015.

[148] M. J. Cooke, M. Stojkovic, and S. A. Przyborski, "Growth of teratomas derived from human pluripotent stem cells is influenced by the graft site," Stem Cells and Development, vol. 15, no. 2, pp. 254-259, 2006.

[149] Q. Zhao, C. A. Gregory, R. H. Lee et al., "MSCs derived from iPSCs with a modified protocol are tumor-tropic but have much less potential to promote tumors than bone marrow MSCs," Proceedings of the National Academy of Sciences of the United States of America, vol. 112, no. 2, pp. 530-535, 2015.

[150] S. Yasuda, S. Kusakawa, T. Kuroda et al., "Tumorigenicityassociated characteristics of human iPS cell lines," PLoS One, vol. 13, no. 10, article e0205022, 2018.

[151] J.-H. Lee and S.-J. Seo, "Biomedical application of dental tissue-derived induced pluripotent stem cells," Stem Cells International, vol. 2016, Article ID 9762465, 7 pages, 2016.

[152] M. Wernig, A. Meissner, J. P. Cassady, and R. Jaenisch, “cMyc is dispensable for direct reprogramming of mouse fibroblasts," Cell Stem Cell, vol. 2, no. 1, pp. 10-12, 2008.

[153] M. Silva, L. Daheron, H. Hurley et al., "Generating iPSCs: translating cell reprogramming science into scalable and robust biomanufacturing strategies," Cell Stem Cell, vol. 16, no. 1, pp. 13-17, 2015.

[154] J. Yu, M. A. Vodyanik, K. Smuga-Otto et al., "Induced pluripotent stem cell lines derived from human somatic cells," Science, vol. 318, no. 5858, pp. 1917-1920, 2007.

[155] K. Kim, A. Doi, B. Wen et al., "Epigenetic memory in induced pluripotent stem cells," Nature, vol. 467, no. 7313, pp. 285290, 2010.

[156] J. M. Polo, S. Liu, M. E. Figueroa et al., "Cell type of origin influences the molecular and functional properties of mouse induced pluripotent stem cells," Nature Biotechnology, vol. 28 , no. 8, pp. 848-855, 2010.

[157] L. G. Villa-Diaz, A. M. Ross, J. Lahann, and P. H. Krebsbach, "Concise review: the evolution of human pluripotent stem cell culture: from feeder cells to synthetic coatings," Stem Cells, vol. 31, no. 1, pp. 1-7, 2013.

[158] G. A. Erickson, S. R. Bolin, and J. G. Landgraf, "Viral contamination of fetal bovine serum used for tissue culture: risks and concerns," Developments in Biological Standardization, vol. 75, pp. 173-175, 1991.

[159] T. Takeda-Kawaguchi, K. Sugiyama, S. Chikusa et al., "Derivation of iPSCs after culture of human dental pulp cells under defined conditions," PLoS One, vol. 9, no. 12, article e115392, 2014.

[160] J. Gorecka, V. Kostiuk, A. Fereydooni et al., "The potential and limitations of induced pluripotent stem cells to achieve wound healing," Stem Cell Research \& Therapy, vol. 10, no. 1, p. $87,2019$.

[161] J. Bilic and J. C. Izpisua Belmonte, "Concise review: induced pluripotent stem cells versus embryonic stem cells: close enough or yet too far apart?," Stem Cells, vol. 30, no. 1, pp. 33-41, 2012.
[162] A. Colman and O. Dreesen, "Induced pluripotent stem cells and the stability of the differentiated state," EMBO Reports, vol. 10, no. 7, pp. 714-721, 2009.

[163] N. Maherali and K. Hochedlinger, "Guidelines and techniques for the generation of induced pluripotent stem cells," Cell Stem Cell, vol. 3, no. 6, pp. 595-605, 2008.

[164] A. Haase, W. Glienke, L. Engels et al., "GMP-compatible manufacturing of three iPS cell lines from human peripheral blood," Stem Cell Research, vol. 35, article 101394, 2019.

[165] L. G. Villa-Diaz, S. E. Brown, Y. Liu et al., "Derivation of mesenchymal stem cells from human induced pluripotent stem cells cultured on synthetic substrates," Stem Cells, vol. 30, no. 6, pp. 1174-1181, 2012. 